\title{
Progress and potential in organoid research
}

\author{
Giuliana Rossi ${ }^{1,3}$, Andrea Manfrin ${ }^{1,3}$ and Matthias P. Lutolf ${ }^{1,2 *}$
}

\begin{abstract}
${ }^{1}$ Laboratory of Stem Cell Bioengineering, Institute of Bioengineering, School of Life Sciences and School of Engineering, EPFL, 1015 Lausanne, Switzerland.

${ }^{2}$ Institute of Chemical Sciences and Engineering, School of Basic Science, EPFL, ${ }^{1015}$ Lausanne, Switzerland.

${ }^{3}$ These authors contributed equally

*Email: matthias.lutolf@epfl.ch
\end{abstract}

\begin{abstract}
Tissue and organ biology are very challenging to study in mammals, and progress can be hindered, particularly in humans, by sample accessibility and ethical concerns. However, advances in stem cell culture have made it possible to derive in vitro 3D tissues called organoids, which capture some of the key multicellular, anatomical and even functional hallmarks of real organs at the micrometre to millimetre scale. Recent studies have demonstrated that organoids can be used to model organ development and disease and have a wide range of applications in basic research, drug discovery and regenerative medicine. Researchers are now beginning to take inspiration from other fields, such as bioengineering, to generate organoids that are more physiologically relevant and more amenable to real-life applications.
\end{abstract}

Over the past few decades, a large body of work generated by developmental and stem cell biologists has provided an understanding of how to control stem and progenitor cell behaviours, such as self-renewal and differentiation along specific tissue lineages, at a molecular level. At the same time, research in the field of regenerative medicine has shown that organs can be repaired by dissociated stem cells that differentiate into one or more of the required mature cell types ${ }^{1}$. Taken together, these developments helped to cultivate the idea that stem cells could be used to re-create organs in vitro. Indeed, the past decade has seen a marked shift towards the use of organoids for studying tissue and organ biology ${ }^{2-4}$. Nevertheless, the history of organoids could be considered to date back to the 1970s, when Howard Green and his colleagues demonstrated that co-cultures of primary human keratinocytes and 3T3 fibroblasts form stratified squamous epithelial colonies resembling the human epidermis, with cell proliferation in the basal layers and keratinization in the upper layers ${ }^{5}$. However, similar to traditional mammalian cell culture, this and many subsequent approaches involved growing cells on artificial 2D surfaces, which precludes the study of behaviours that are intrinsically linked to the 3D organization of cells into tissues and organs. The establishment of 3D culture systems, which resulted from a better understanding of extracellular matrix (ECM) biology $\mathrm{y}^{6,7}$ and the development of methods for culturing cells in suspension ${ }^{8,9}$, played a key part in enabling organoid culture. Among the first examples of what today might legitimately be considered an organoid were structures derived from the 
mammary gland by Mina Bissell and colleagues, which showed that in vitro morphogenesis could be observed in 3D laminin-rich matrices ${ }^{10,11}$. These cultures resulted in functional alveolus-like structures characterized by epithelial polarization and directional secretion, which developed by virtue of their interaction with the basement membrane ${ }^{10}$. However, it was perhaps the groundbreaking work by the groups of Yoshiki Sasai ${ }^{12}$ and Hans Clevers ${ }^{13}$ that kick-started the organoid field. Clevers and co-workers demonstrated that intestinal organoids $^{13}$ could be derived from intestinal adult stem cells (ASCs) whereas Sasai and colleagues showed that pluripotent stem cells (PSCs) could be used to recapitulate in vitro the development of cortical tissues ${ }^{14}$ and the optic cup ${ }^{12}$. Their work promoted the idea that stem cells have the intrinsic ability to self-organize into 3D structures that resemble in vivo organs, which remains one of the cardinal concepts underlying organoid biology. Subsequent to these pioneering studies, other scientists have succeeded in generating organoids from a wealth of different cellular sources and from different species (Table 1).

Over the years, the term organoid has been used to define different types of in vitro cultures, from tissue explants ${ }^{15-17}$ to organ-on-chip systems ${ }^{18}$, with the general meaning being 'similar to an organ'. However, this broad definition may lead to confusion ${ }^{19}$. In this Review, we consider organoids to be complex 3D structures that display architectures and functionalities similar to in vivo organs and that develop from stem cells or organ-specific progenitors through a self-organization process ${ }^{2,3}$. Here, we describe the main classes of organoids and how they can be derived and discuss their existing and emerging applications in the fields of basic biology, disease modelling and regenerative medicine. We highlight some of the key challenges that the field is encountering and provide a forward-looking framework for how multidisciplinary approaches could be used to build next-generation organoid platforms. In particular, we take inspiration from tissue engineering ${ }^{20}$, a field classically focused on the design and fabrication of biocompatible materials that serve as cell-instructive scaffolds for tissue reconstruction. Tissue engineers have conceived a wide range of technologies to augment tissue regeneration that could further improve organoid cultures. We do not discuss in detail specific organoid types or their applications in specific fields and instead refer interested readers to several excellent recent reviews on these topics ${ }^{21-26}$.

\section{Deriving organoids}

Organoid development usually involves the self-organization of a fairly homogeneous cell population. Selforganization can be defined as the capacity of a cellular system that initially lacks an ordered structure to spatially rearrange under the guidance of system-autonomous mechanisms, even if exposed to a uniform signalling environment ${ }^{4}$. Much of what is known about this process comes from decades of research in the field of developmental biology. Conceptually, the self-organization process can be broken down into self-patterning events and morphogenetic rearrangements ${ }^{4}$.

Self-patterning is the formation of patterns of cell differentiation in an initially homogeneous system that result from system-autonomous mechanisms and local intercellular communication. Self-patterning usually starts with a symmetry-breaking event ${ }^{4,27}$, for which interplay between a number of different mechanisms has been proposed, including reactiondiffusion mechanisms ${ }^{28,29}$, bistability of regulatory networks ${ }^{30}$ and asymmetric cell division ${ }^{4}$. Many of these mechanisms rely on positive and negative feedback circuits and crosstalk between them ${ }^{28-30}$. Morphogenetic rearrangements result from sorting of different cell types 
within a tissue and from higher-level reorganization of the system's architecture. Cell sorting is mediated by physical interactions between different cell types, which involve differences in cell-cell adhesion, cortical tension and/or contractility and cell motility ${ }^{4,31}$. Architectural rearrangements are sustained by system-intrinsic mechanics resulting from changes in cell shape, cell contraction, cell movement or differential tissue expansion ${ }^{4}$. Successful derivation of organoids relies on recapitulating these processes and requires careful consideration of three main features: the physical characteristics of the culture environment; the requirement for system-autonomous (that is, endogenous) and/or exogenous signals; and the starting cell type and system conditions. Choices made regarding each of these features can affect the characteristics of the final organoid.

\section{Physical characteristics of the culture environment.}

The most common way to promote the 3D characteristic of organoids is to use solid ECMs that support cell growth and to which cells can adhere (Fig. 1a). Matrigel, a natural ECM purified from Engelbreth-Holm-Swarm mouse sarcoma7, is the most widely used matrix for $3 D$ organoid derivation. Intestinal ${ }^{13}$, cerebral ${ }^{32}$, gastric ${ }^{33}$ and mammary gland ${ }^{17}$ organoids are only some of the examples that have been successfully generated using Matrigel or similar animal-derived hydrogels that mimic basement membranes. There have also been some rare examples of the use of collagen type I matrices for derivation of mammary gland ${ }^{34}$ and intestinal $\left.\right|^{35}$ organoids. The main advantage of these natural matrices is the presence of a complex mix of ECM components and growth factors, which makes cell growth and differentiation very efficient. However, this complexity and the variability in composition make it more difficult to control the culture environment and may lower reproducibility. For these reasons, chemically defined hydrogels have been recently introduced to support intestinal ${ }^{36}$ and cerebral ${ }^{37}$ organoid culture as a substitute for the less well-defined natural matrices. These hydrogels allow the biochemistry and mechanics of the culture environment to be controlled but are inherently less bioactive and need to be customized to match the specific requirements of different organoids.

A different strategy, which has been adopted for the derivation of optic cup ${ }^{12}$, cerebral ${ }^{14}$, cerebellar ${ }^{38}$ and hippocampal ${ }^{39}$ organoids, is the culture of 3D cell aggregates in suspension (Fig. 1a). Although the suspension culture approach does not make use of a solid scaffold for cell embedding, in some cases low concentrations of Matrigel are added to promote the formation of polarized epithelial structures ${ }^{12}$.

Kidney organoids ${ }^{40,41}$ have been generated using an air-liquid interface method in which cells are cultured in the form of a pellet on a thin, microporous membrane, with cell culture medium only on the basal side of the membrane (Fig. 1a). Cell pellets subsequently selforganize into a multi-layered structure that resembles the microarchitecture of the native kidney.

At this point in time, the decision to use a specific organoid derivation protocol is largely driven by empirical considerations. Indeed, systematic comparisons of different protocols for deriving a specific organoid type are lacking, which means it is not yet possible to appreciate the relative strengths, weaknesses and applications of each protocol.

\section{Endogenous and exogenous signals.}

Most organoids are derived from a starting cell population that has been exposed to specific morphogens at defined time points, which results in the activation of desired developmental signalling pathways. In turn, these signals can trigger self-organization, but only if all the 
necessary components are present in the system: missing components must be provided exogenously for self-organization to proceed appropriately.

The derivation of some organoids relies almost exclusively on endogenous signals (Fig. 1C). For example, mouse optic cup organoids are derived from mouse PSCs that are aggregated and cultured in a serum-free medium containing low levels of growth factors. These conditions support the formation of a uniform neuroepithelium (NE), after which selfpatterning mechanisms specify spatially separated domains of neural retina (NR) and retinal pigmented epithelium (RPE) (Fig. 1C). Morphogenesis then also proceeds without exogenously provided signals ${ }^{12}$. Thus, the starting cell population already possesses all the required components to self-organize into an optic cup, likely following its default developmental trajectory. Nevertheless, it should be noted that although no additional factors are supplied during derivation of these organoids, it is possible that a small quantity of exogenous signals could be present in the minimal amount of Matrigel used in the procedure.

The majority of organoid derivation protocols do require that specific exogenous signals are provided because the initial cellular system does not contain all the essential components to undergo the desired self-organization process. In some cases, exogenous signals are necessary only for induction of initial cell types, which then rely on system-autonomous signals to undergo the remaining steps of self-organization. For example, human PSCs (hPSCs) must be exogenously stimulated with specific growth factors to form a mixed population of ureteric epithelium and metanephric mesenchyme cells ${ }^{40}$, which will then self-organize into kidney organoids without addition of further factors to the medium ${ }^{25,40}$ (Fig. 1c).

Finally, for many organoids, the cellular system must be stimulated with appropriate and specific exogenous signals throughout the derivation process. hPSC-derived gastric organoids are just one of several such examples: exogenously supplied factors are necessary to push hPSC-derived definitive endoderm cells towards a posterior foregut fate ${ }^{33}$ (Fig. 1C); further exogenous stimulation is then required to guide cells towards an antral or fundic gastric epithelial fate and to direct growth, morphogenesis and differentiation into functional gastric cell types ${ }^{33,42}$.

Although these considerations apply to all types of organoids, PSC-derived organoids in particular often require timed exposure to specific factors to guide the system through the desired developmental trajectories. The decision on which specific factors to use, and when they are applied, is generally informed by the current understanding of relevant in vivo developmental mechanisms.

\section{Starting cell type and initial culture conditions.}

Protocols for the derivation of different organoids vary in the starting state of the cell population (Fig. 1b). Depending on the initial conditions, a cell ensemble will undergo all or only some of the steps involved in self-organization. When organoids are derived from a single cell type (as is the case for optic cup or small intestine organoids), self-organization of the cell population necessarily involves symmetry-breaking and subsequent patterning to generate spatially separated domains of different cell types. This patterned structure can then undergo morphogenetic rearrangements to acquire the final organoid architecture. In general, when an organoid derivation protocol starts from a single cell, an early step of cell expansion is required before self-organization can occur. At the opposite end of the spectrum, there are protocols that involve the co-culturing of cell types that have been separately predifferentiated, which is the case for PSC-derived liver organoids ${ }^{43}$. In these protocols, the 
disparate cell identities are to a large extent already established; therefore, self-organization primarily involves cell sorting and subsequent architectural rearrangements (Fig. 1b). The initial conditions of the cell population will also affect the range of applicability of an organoid as a biological model system. Indeed, organoids obtained through the co-culture of separately pre-established cell types are arguably less informative for studying organogenesis than models in which the different cell types are generated simultaneously and therefore have the potential to capture more comprehensively the transient developmental interactions that could occur between different progenitors during the formation of the organoid.

The characteristics of the final organoid also depend on the starting cell type. Organoids have been derived from ASCs ${ }^{13,17,34,44}$ (either as isolated cells or from dissected tissue fragments), $\mathrm{PSCs}^{12,32,33,45}$ or fetal progenitor cells ${ }^{46,47}$. Some types of organoids have thus far been derived only from PSCs, including neuroectodermal organoids, such as optic cup ${ }^{12}$ and cerebral organoids $^{14,32}$, and mesodermal kidney organoids ${ }^{40,48}$. By contrast, organoids of surface ectoderm lineage (particularly glandular tissues) have been mainly derived from ASCs or dissociated adult tissues ${ }^{34,49-51}$. The majority of organoids of the endodermal lineage have been derived from both PSCs and ASCs (Table 1). Terminally differentiated cells reprogrammed to a tissue-specific ASC-like state have recently been used as an alternative source for derivation of epithelial mammary gland and pancreatic organoids ${ }^{52}$. Only a few examples of organoids derived from fetal progenitors have been published. Pancreatic organoids have been derived using cells dissociated from mouse fetal (embryonic day 10.5) dorsal pancreata ${ }^{47}$ and fetal-type intestinal organoids have been obtained from dissociated human fetal intestinal tissue ${ }^{46}$.

Because different cell types originate from distinct developmental stages, they will follow different paths Fig. 2); therefore, the choice of the starting cell population is particularly important when studying mechanisms underlying organoid formation. Organoids derived from ASCs or adult tissue fragments are believed to mimic the homeostatic or regenerative conditions of their tissue of origin ${ }^{2}$. Thus, stem cells from organs that are characterized by continuous renewal throughout life, such as the epithelia of the small intestine ${ }^{13}, \operatorname{colon}^{53}$ or stomach $^{54,55}$, generate organoids representing the homeostatic role of these cells in vivo. By contrast, organoids derived from tissues with a slow turnover ${ }^{2}$ in which endogenous stem and/or progenitor cells presumably play a role only upon injury, such as in the pancreas or liver, are considered to be bona fide models of regeneration ${ }^{56,57}$. Although this classification is clear in vivo, in vitro conditions can make the distinction between homeostasis-driven and regeneration-driven organoids tricky to determine. For example, it has been reported that intestinal organoid formation occurs through mechanisms that seem to be unnecessary for homeostasis in vivo but are activated under regenerative conditions ${ }^{58}$. Similarly, gastric organoids can be obtained from a starting population of cells that specifically expands in vivo upon injury ${ }^{44}$.

While ASC-derived organoids are useful for answering questions about adult tissue biology, PSC-derived organoids are mainly used to study organogenesis and developmental events that lead to tissue formation (Fig. 2). PSC-derived organoids very rarely reach an adult tissue stage in vitro and usually resemble fetal-stage tissues ${ }^{12,33,45}$ (Fig. 2). One possible reason for the limited maturation of PSC-derived organoids is the fact that development towards more mature cell types requires continuous culture for periods of time that typically exceed the capacity of the actual culturing methodologies ${ }^{59}$. Another possibility is that, in contrast to ASC-derived organoids, cells in PSC-derived organoids are missing crucial interactions with 
other co-developing cell types because of our limited capacity to fully reconstruct embryonic developmental processes in vitro ${ }^{60}$.

So-called embryonic organoid systems can be used to build more complete developmental models in vitro. These self-organizing PSC-derived structures, also termed 'embryoids' or 'gastruloids', mimic in a very simplified way pre-implantation ${ }^{61}$ and early post-implantation embryonic development ${ }^{62-64}$, establishment of body axes ${ }^{65,66}$, gastrulation ${ }^{65-71}$ and neural tube development ${ }^{72,73}$. Whereas classic organoids are typically composed of a restricted subset of cell types from one germ layer, embryonic organoids are characterized by the coexistence of cells from multiple germ layers, as in real embryos. This feature adds a fascinating level of complexity and allows for the reproduction and in vitro study of the intricate interactions among different cell types in developmental processes.

Finally, fetal progenitor-derived organoids ${ }^{46,47}$ start directly from fetal tissues, thus from a stage between ASCs and PSCs. Therefore, fetal organoids can be used to study more advanced stages of organogenesis than PSC-derived organoids. For example, enterospheres from fetal intestinal progenitors have been used to study their maturation towards adult intestinal organoids ${ }^{46}$.

\section{Applications of organoids}

\section{Organoids as models of development, homeostasis and regeneration.}

Organoids recapitulate in vitro some principles of organ biology and provide simplified and readily accessible 'minimal systems' for discerning the relative contribution of different tissue components to complex morphogenetic processes (Fig. 3a). Indeed, organoids have already revealed surprising mechanisms of tissue-autonomous self-organization in homeostasis, regeneration and development. These tissue-autonomous mechanisms would have been difficult to identify in vivo because of the potentially confounding influences of nearby tissues. For instance, in ASC-derived intestinal organoids, stem cells isolated from crypts re-establish an intestinal epithelium in the absence of a subepithelial mesenchymal compartment ${ }^{13}$, and in PSC-derived optic cup organoids, optic vesicles autonomously undergo morphogenesis without the need for signals from an overlying surface ectoderm ${ }^{12}$.

A key feature of organoids is their greater experimental accessibility than mammalian animal models, which has contributed to a more in-depth understanding of organogenesis and adult organ biology. The capacity of mouse intestinal stem cells (ISCs) that express the $\mathrm{Lgr}^{5}$ gene to form self-renewing colonies has been shown to be dependent on Paneth cells, a neighbouring cell type of ISCs in the crypt ${ }^{74}$. Indeed, Paneth cells express high levels of WNT ligands, and WNT signalling is essential for ISC maintenance ${ }^{75}$. An elegant study used cells expressing a tagged WNT isoform to gain insights into the role of WNT gradients in intestinal biology ${ }^{76}$. The use of intestinal organoids in this study provided an experimentally amenable system to generate high-resolution information ${ }^{76}$. This work demonstrated that the WNT protein does not diffuse but is instead transferred from a Paneth cell to an adjacent ISC, and the WNT gradient is formed along the crypts by ISC division and dilution of membrane-bound WNT in daughter cells. Taken together, these observations indicate that Paneth cells act as localized sources of WNT signals for ISC self-renewal ${ }^{76}$ and support a model in which spatial confinement of Paneth cells to the bottom of the crypt defines the physical separation between ISC self-renewal and differentiation. This physical separation was previously suggested by clonal analysis experiments on ISCS in vivo, which demonstrated that 
competition for occupancy of the crypt niche determined whether ISCs self-renewed or differentiated ${ }^{77}$.

Similarly, experiments possible only on optic cup organoids have provided insights into the mechanisms underlying optic cup morphogenesis, a process that involves invagination of the NR within the RPE. Treating mouse organoids at different phases of the conversion from optic vesicle to optic cup with drugs that inhibit either active cytoskeletal contraction or cell proliferation showed that optic cup morphogenesis is a multi-phase process. The process of vesicle invagination starts with domain-specific changes to mechanical properties, which are caused by cytoskeletal re-organization. These changes result in a reduction of contractility and thus rigidity in the NR and maintenance of high levels of contractility and rigidity in the RPE. In subsequent phases, the role of cell proliferation becomes or differences between humans and other species. For example, mouse embryos lacking the $\beta$-catenin gene, which encodes the core transcription factor of the WNT pathway, show a loss of fundic stomach identity and an expansion of the antral domain ${ }^{42}$. This result suggests a prominent role for WNT signalling in specifying fundic identity. To determine whether this might also be the case in human gastric patterning, hPSC-derived gastric organoids ${ }^{33}$ at the posterior foregut stage were exposed to a WNT agonist, and expression analysis of domain-specific markers confirmed a switch from antral to fundic identity ${ }^{42}$.

Whereas gastric organoids highlight common developmental mechanisms between humans and mice, optic cup organoids underline human-specific developmental features ${ }^{12,78}$. Indeed, human optic cup organoids take much longer than their mouse counterparts to reach comparable developmental stages. They are also larger and have a thicker NR than mouse organoids. These species-specific differences are confirmed by the analysis of human and mouse embryonic tissues ${ }^{78}$. Moreover, optic cup organoids have revealed species-specific morphogenetic mechanisms. Normally, during the progression from optic vesicle to cup, the NR changes from an apically concave to an apically convex structure. In mouse organoids, this so-called eversion requires continuity between the NR and the RPE. Indeed, mouse NRs dissociated from the RPE at the vesicle stage do not undergo eversion ${ }^{12,78}$. By contrast, human NRs dissociated from the RPE at the vesicle stage are still able to autonomously undergo eversion through a myosin-independent mechanism mediated by integrins ${ }^{78}$.

Finally, organoids can be used as minimal systems to study the principles of self-organization. An interesting example comes from research on embryonic organoid models of gastrulation (gastruloids) ${ }^{66-71}$. In these systems, cells adopt spatially organized fates as a result of uniform exposure to a morphogen, which implies they self-organize ${ }^{65,67,69-71}$. In some of these studies, a combination of in silico mathematical models and experimental data has been used to test the capacity of specific molecular mechanisms to recapitulate the outcome of selforganization ${ }^{67,70}$. These models were then used to successfully predict the behaviour of gastruloids in response to varying the starting conditions ${ }^{67,70}$. In general, mathematical modelling could help to elucidate the mechanisms underlying self-organization and to predict the effect of different conditions on the outcome, thus increasing our ability to control complex multicellular behaviours in vitro.

\section{Organoids for disease modelling, drug discovery and personalized medicine.}

An obvious advantage of organoid cultures for disease modelling, compared with traditional cell cultures of a single cell type, is their ability to mimic pathologies at the organ level (Fig. 3b). Moreover, organoids derived from human ASCs or induced PSCs (iPSCs) can serve as models for human diseases by recapitulating specific human features that are relevant for 
translational studies. A wide range of organoid-based disease models that reproduce genetic diseases ${ }^{32,79-81}$, host-pathogen interactions ${ }^{33,55,82-94}$ or cancer ${ }^{21,55,95-98}$ have already been developed and provide proof of principle that organoids can reproduce certain well-known pathological features (Fig. 3b). For example, human gastric organoids microinjected with Helicobacter pylori bacteria successfully reproduce the typical signs of this bacterial infection 33,55 . This model is particularly relevant because species-specific features of the stomach make animal models unsuitable for studying human gastric pathologies. Indeed, $\mathrm{H}$. pylori infection in mice does not progress to ulceration and cancer as it does in humans ${ }^{55}$.

The idea that organoids can model human pathologies has opened the door to studies on the feasibility of drug testing and screening applications (Fig. 3c). For instance, drugs for treating Zika virus infections were recently screened in hPSC-derived cortical neural progenitor cells and validated in parallel in organoids and mouse models, confirming that organoids are a valid alternative system for testing drugs against this pathology ${ }^{99}$. Pioneering results have also been reported for the use of organoids in testing drugs for cystic fibrosis (CF), a genetic disease caused by defects in the CFTR gene ${ }^{100}$. Patient-derived intestinal organoids carrying CFTR mutations possibly represent the only example of organoids that have already been applied to personalized medicine (Fig. $3 \mathrm{~d}$ ). Organoids derived from one patient with a very rare CFTR mutation with no known treatment were used to screen existing CF drugs. They showed responsiveness to a drug already used to treat other CF mutations, thereby allowing the patient access to treatment with beneficial outcomes ${ }^{101}$. The screen used a standardized functional test based on CAMP-induced organoid swelling as a readout for restored CFTR functionality, which was optimized on intestinal organoids from both mice and patients for at least five different mutations ${ }^{79}$. Another milestone in the use of organoids for drug screening is the development of organoid-based high-throughput screening (HTS) paradigms. An interesting example of HTS has been recently reported for human kidney organoids ${ }^{102}$, in which automated organoid derivation and assessment of drug effects have been demonstrated.

An emerging application for organoids is the creation of organoid biobanks for different pathologies (Fig. 3c). Such biobanks will eventually facilitate the design of powerful screening platforms that cover a wide range of the worldwide population genetic variance. For example, the creation of CF intestinal organoid biobanks ${ }^{103}$ could potentially cover most of the spectrum of CFTR mutations. The generation of biobanks could be even more important for cancer, a class of pathologies that are characterized by a virtually unlimited number of mutations ${ }^{104,105}$. Cancer cells have been used to generate in vitro structures in a similar fashion to organoids. However, these structures, just like the tumours from which they are derived, often lack the typical architectural organization of organoids ${ }^{21,53,95-97}$. These models are usually defined as cancer organoids or, perhaps more appropriately, 'tumouroids'. Initial attempts have been made to establish tumouroid biobanks for colon cancer ${ }^{104,105}$, which is very common in humans. One study generated a biobank of primary colon cancer organoid lines from ${ }^{20}$ patients that preserved the histopathological and main genetic features of the original tumours. Furthermore, a proof-of-concept drug screen showed that the drug responses of organoids with different mutations matched prior clinical observations ${ }^{105}$. Another group developed a biobank of ${ }^{55}$ different colorectal tumouroids representing different histological subtypes and clinical stages ${ }^{104}$. The number of tumouroid biobanks is rapidly increasing: a biobank of gastrointestinal cancer organoids was recently used to compare patient clinical responses to drugs with in vitro responses ${ }^{106}$, thereby highlighting the predictive potential of cancer organoids, and a library of ${ }^{22}$ patient-derived bladder cancer 
organoids has been established that recapitulate the histopathological and molecular hallmarks of the respective parental tumours ${ }^{107}$.

Organoids from patient-derived iPSCs could also be used to study pathologies for which defined genetic causes have not yet been discovered. Complex pathologies with high patientto-patient variability might be good candidates for these studies. Indeed, new biological insights into autism spectrum disorder (ASD) were recently generated by modelling ASD in brain organoid cultures; this approach uncovered in part a pathogenic mechanism that involves overproduction of GABAergic neurons in response to FOXG1 overexpression ${ }^{108}$ (Fig. 3b).

All these results are highly encouraging and suggest that organoids could reduce the amount of required animal testing in accordance with the $3 R$ principles ${ }^{109}$.

\section{Regenerative medicine.}

Organoids are emerging as a promising source of transplantable tissues and functional cell types for cell therapy in regenerative medicine (Fig. 3e). Proof-of-concept experiments have already been reported in animal models. For example, retinal sheets have been derived from mouse embryonic stem cells (ESCs) or mouse iPSCs using a modified optic cup organoid protocol $^{12}$ and transplanted into a mouse model of retinal degeneration. The transplanted tissue gave rise to mature photoreceptors and, in some cases, established synaptic connections with the host cells ${ }^{110}$ and recovered light responsiveness ${ }^{111}$. Retinal tissues generated from human ESC-derived organoids also survive, mature and demonstrate a degree of integration with the host tissue when transplanted into rat and primate models of retinal degeneration ${ }^{112}$. Intestinal organoids grown from dissociated mouse colon epithelia or from single stem cells and transplanted into mice were able to regenerate colonic mucosa injuries to various degrees ${ }^{113}$. Similarly, transplanted enterospheres grown from fetal intestinal progenitor cells integrate into injured mouse colon and differentiate in vivo ${ }^{46}$. Transplantation experiments in animal models have also been reported for liver ${ }^{57,80}$ and kidney ${ }^{48}$ organoids. Indeed, the ability of organoids to provide cells that are competent to reconstruct organ function and potentially also a niche that protects the graft from the hostile pathological environment could represent an advantage in cell therapy applications compared with transplantation of cells deprived of their physiological context. This hypothesis is supported by a study that reported that 3D-structured grafts integrate into the host mouse retina better than dissociated embryonic retinal progenitors ${ }^{110}$.

Finally, the use of organoids for regenerative medicine could be used in combination with in vitro genetic correction strategies to enable the autologous replacement of tissues affected by genetic disorders. In a proof-of-principle study, CRISPR-Cas9-mediated gene editing was used to correct the most common CFTR mutation leading to CF, a deletion of phenylalanine at position ${ }^{508}$, in patient-derived ISCs, which were then used to generate functional organoids $^{114}$ (Fig. 3e).

Although preliminary, taken together, these results are highly promising and indicate that the clinical transplantation of organoid-derived cells and tissues might be feasible. 


\section{Towards a new generation of organoids}

The applications discussed above rely on the reproducible generation of organoids with a high level of structural and functional similarity with real organs to be used as faithful substitutes for in vivo studies. Although the organoid field is moving ahead at an impressive speed, bridging the gap with native organs will be the main challenge in the decades to come. A general limitation of all organoid derivation protocols is the high variability of the phenotypes that they can produce. Organoid-to-organoid reproducibility is a limitation for many applications but is particularly problematic for translational studies, such as drug screening, in which a high intrinsic variability could mask the effect of a treatment. Moreover, different types of organoids recapitulate with different accuracy the cellular and architectural complexity of their respective organ. This characteristic applies to the capacity of an organoid to reproduce all cell types within a specific tissue as well as the different tissues of an organ. For example, whereas intestinal organoids from ASCs are exclusively composed of intestinal epithelium ${ }^{13}$, derivation of intestinal organoids from PSCs can also give rise to the intestinal mesenchyme ${ }^{45}$. Another important limitation, especially for PSC-derived organoids, is their low grade of maturation, which hinders their application as models for adult tissue biology. Below, we discuss how progress in other disciplines could help overcome these shortcomings (Fig. 4).

\section{In-depth characterization of organoids.}

Organoids are complex structures composed of different cell types arranged in 3D space but, for most systems, the identity of these cell types has not been thoroughly defined. This makes it difficult to determine how accurately an organoid represents native organ composition and architecture. Single-cell sequencing technologies provide a powerful way to relate the transcriptional profiles of organoids to those of targeted organs. This approach not only provides information on cellular identities within organoids but also can help to define the lineage trajectories of selected cell types. This type of analysis has already been conducted for cerebral organoids ${ }^{115,116}$ and liver organoids ${ }^{117}$, which have been shown to have similar gene expression profiles to fetal neocortex and fetal liver, respectively. As a result of a multilaboratory project, stage-dependent transcriptome data from embryonic animal tissues are becoming available ${ }^{118}$, and this information will help reveal how faithfully organoids recapitulate developmental processes. Moreover, RNA tomography can generate cell transcriptional profiles with spatial resolution, thereby providing information on the position of different cell types within the organ architecture ${ }^{119}$. These impressive early efforts towards a deeper characterization of organoids will ultimately also contribute to the improved reproducibility of organoid cultures.

\section{Improved organoid maturation.}

As discussed above, a major shortcoming of PSC-derived organoids is their limited cell maturation. Indeed, organoids of this type usually resemble fetal tissues rather than adult ones. On the one hand, this might simply be due to the restricted lifespan of organoids, which limits their ability to reach later stages of development. On the other hand, it is possible that in vitro conditions lack specific in vivo environmental factors that promote organoid maturation. For example, brain organoids could require sensory stimulation for further maturation, which contributes to the shaping of neural circuits in vivo ${ }^{120}$. Lengthening the 
culture period is particularly crucial for human organoids because they take much longer than mouse organoids to reach comparable stages ${ }^{12,78}$.

An obvious reason for the limited lifespan of organoids is that, once a certain size is reached, diffusion is unable to supply all the cells with sufficient nutrients to support continued development ${ }^{90}$. A possible solution to this problem could be the use of bioreactors that improve nutrient supply through constant culture spinning (Fig. 4a). Bioreactors are extensively applied in the field of tissue engineering to introduce controlled changes of culture conditions, standardize them and scale up tissue production for regenerative medicine ${ }^{121,122}$. Indeed, such bioreactors have extended the duration of brain organoid cultures from a couple of months to up to 1 year ${ }^{123}$ and have resulted in structures that more closely resemble the developing human brain.

Vascularization of organoids could further improve their lifespan by distributing nutrients via capillaries, as occurs in vivo (Fig. 4b). Emerging bioengineering approaches that have been developed to generate vascularized tissue-like structures include sacrificial moulds ${ }^{124-126}$ and laser ablation ${ }^{127}$, which enable fabrication of channels within the culturing scaffold that can host endothelial cells and form perfusable vascular units. Alternatively, bioprinting methods ${ }^{128,129}$ have been used to control the position of endothelial cells in 3D-printed structures. Although these studies were conducted in simplified cellular systems, organoid culture could benefit from future improved versions of these methods.

Another strategy to promote vascularization of the growing tissue would be to integrate endothelial cells, or their progenitors, during organoid formation. This approach has been successfully applied to liver organoids: human endothelial cells were co-cultured with human mesenchymal stem cells and human iPSC-derived hepatic endoderm cells to form selforganizing liver buds with a microvascular network that rapidly connected to the host circulation upon transplantation into mice ${ }^{43}$ (Fig. 4f). Alternatively, the desired organ-specific cells and vascular endothelial cells can be independently differentiated from PSCs and the endothelial cells then used to vascularize the organoid, as has been shown for human brain organoids ${ }^{130}$. Recently, it has been demonstrated that hPSC-derived cerebral organoids implanted into mouse brains can be vascularized by the host ${ }^{131}$. Interestingly, the hostvascularized organoid grafts survive for up to ${ }^{233}$ days post-implantation and reach later stages of development than do stage-matched cerebral organoids cultured in vitro. They also have a higher number of mature neurons, mature astrocytes, microglia and cells expressing markers of dorso-cortical identity ${ }^{131}$. That organoids can acquire a more developed phenotype when transplanted in vivo has also been demonstrated for other organoids, such as liver ${ }^{43}$, intestine ${ }^{132}$ and lung ${ }^{133}$, which suggests that exposure to in vivo systemic factors and vascularization promotes the growth and maturation of organoids. Nevertheless, full maturation of some tissues may require many years in vivo, such as the cerebral cortex, which continues to develop postnatally. The full development of such adult traits may require innovative strategies that artificially accelerate maturation processes. For organoids obtained from patient iPSCs, the modelling of age-related phenotypes is even more challenging. Indeed, reprogramming of adult cells tends to erase the original age-related traits ${ }^{134}$. Although not extended to organoids, the transient overexpression of progerin (a truncated form of lamin A) in patient iPSCs has been used to recapitulate age-related traits and has allowed in vitro modelling of pathological features of Parkinson disease ${ }^{134}$. 


\section{Improved organoid architecture.}

Self-organization can generate organoids with a 3D microanatomy that resembles that of in vivo organs, but their overall architecture usually differs greatly from real organs. To address this challenge, stem cell culture scaffolds with a topography matching that of a specific tissue could be used to improve organoid architecture and increase size. For example, microstructured collagen gels that mimic the typical crypt anatomy of the colonic epithelium have been used to culture a self-renewing monolayer of human colonic cells ${ }^{135}$, and an architecture that mimics the crypt-villus structure has been used to culture human small intestinal stem cells ${ }^{136}$. At the microscale, organoid topology could be improved by adopting bioengineering approaches to better regulate self-organization and morphogenesis (for instance, by precisely controlling interactions between stem cells and the surrounding ECM). Most organoid protocols use naturally derived hydrogel matrices such as Matrigel. Although these matrices are extremely effective at promoting cell growth and self-organization, they limit the ability to direct organoid morphogenesis because they are ill-defined, suffer from lot-to-lot variability and do not support controlled modifications. They also contain animalderived products, which are undesirable for clinical applications owing to risks of immunogen and pathogen transfer. To overcome these limitations, a wide range of synthetic, chemically defined hydrogels have been developed for 3D cell culture ${ }^{137-145}$, the chemical and physical properties of which can be controlled and optimized for specific purposes. Some of these materials have already proved their compatibility with specific organoids: cerebral organoids have been grown in hyaluronan-based hydrogel $\mathrm{s}^{37}$ and self-organizing neural tube cysts have been grown in poly(ethylene glycol) (PEG)-based hydrogels ${ }^{72,73}$. Furthermore, human intestinal organoids grown on synthetic hydrogels have been successfully transplanted into mouse models of colonic mucosal wounds in proof-of-concept studies ${ }^{146}$. Recently, new hydrogel formulations have been developed for which the biochemical and biophysical properties can be modulated spatially and temporally $36,147,148$ (Fig. 4C), which potentially adds an interesting new layer of control over organoid morphogenesis. Indeed, it has been shown that the mechanical properties of synthetic hydrogels can be dynamically tuned to initially favour ISC expansion and subsequently trigger crypt emergence and give rise to fully developed intestinal organoids ${ }^{36}$ (Fig. 4c).

Other tools used in bioengineering could also be exploited to increase the control over organoid morphogenesis and self-organization but have not yet been applied to studies directly related to organoids. Such tools include hydrogels that permit instantaneous orthogonal modification of their biochemical and mechanical properties ${ }^{147}$, which could be used to dynamically regulate organoid morphogenesis and progressively guide them towards a desired architecture (Fig. 4c). Another example is light-triggered photoablation ${ }^{127}$, which could be used to actively re-adapt the geometry of the matrix to control morphogenetic rearrangements and favour organoid growth. Alternatively, new chemically defined materials that mimic the viscoelastic behaviours of in vivo ECM $^{149-151}$ could improve organoid growth by continuously reacting and re-adapting to morphogenetic processes without the need for external triggers.

The ability to control how cells assemble (for instance, by positioning different cell types in conformations that bias cell type-specific spatial interactions) could also be used to extend the control over organoid self-organization (Fig. 4d). Bioprinting methods developed to preestablish the 3D spatial organization of cells into tissues could fit this purpose ${ }^{152}$, as could the more recently developed DNA-programmed assembly of cells (DPAC) approach, which exploits the complementarity of DNA oligonucleotides ${ }^{153}$. Moreover, DPAC has been used to 
pattern mouse embryonic fibroblast clusters in a gel, allowing for predictable folding of collagen scaffolds ${ }^{154}$, showing its potential to directly influence morphogenetic processes.

Organoid morphogenesis is also likely to be improved by gaining better control over the spatial and temporal distribution of diffusible signalling molecules (Fig. 4e). Indeed, whereas in vivo cell fate patterning is influenced by spatially localized signalling centres, in vitro signalling molecules are typically presented in a uniform fashion. Bioengineering methods that rely on microfluidic devices ${ }^{155-157}$ or on local diffusion from microspheres ${ }^{158}$ have been developed to reproduce gradients of signalling molecules in both $2 D^{156}$ and $3 D$ cultures $^{155,157,158}$. In the future, these technologies could be applied to better control cell fate patterning during organoid development. Alternatively, synthetic biology approaches could be used to re-engineer the genetic programmes underlying self-organization; in a demonstration of its feasibility, synthetic Notch receptors were used to direct mammalian cells into self-organized cell patterns ${ }^{159,160}$.

Finally, mathematical modelling could also help to define which factors play a role in a specific self-organization process ${ }^{67,70}$. As discussed for the case of gastruloids, in silico models could predict the behaviour of an in vitro system upon exposure to specific stimulations ${ }^{67,70}$. This approach could help to identify in silico the best strategies to obtain a desired outcome.

\section{Approaches to improve organoids for disease modelling.}

Another limitation of organoids, particularly for disease modelling applications, is their inability to model multi-organ pathologies. Co-culturing approaches (Fig. $4 \mathrm{f}$ ) could partially address this issue. An early example in this direction has been reported for hPSC-derived intestinal organoids and enteric neurons ${ }^{161}$. In this study, the authors observed that mutations in PHOX2B, a gene associated with Hirschsprung disease, limit the differentiation of the enteric neuronal population, resulting in poor development of the smooth muscle components associated with the intestinal organoid epithelium ${ }^{161}$. For those pathologies with a strong immune component, the inclusion of vascularized networks perfused with immune cells could be a good strategy. Moreover, current drug testing platforms could be improved by merging organoid cultures with organ-on-chip technologies ${ }^{162}$ to generate $3 \mathrm{D}$ systems that re-create the crosstalk between different organs (Fig. $4 \mathrm{~g}$ ). This approach could combine the advantages of the two systems, bridging the ability to have interconnected, minimal systems typical of organs-on-chip with the high in vivo faithfulness and functionality typical of organoids. Clearly, the use of structured organoids requires novel microchip concepts that could seamlessly integrate with the highly stereotypical organization of organoids. Another crucial aspect for drug screening and personalized medicine applications of organoids is the development of reliable assays with accurate readouts of effectiveness, such as the CTFR drug screening assay conducted on intestinal organoids ${ }^{79}$. Integration of biosensors into the culture system could provide dynamic, automated means to quantitatively measure physicochemical responses to drugs and to provide custom readouts for drug screening applications, similar to approaches already demonstrated for organ-on-chip technology ${ }^{163}$.

\section{Conclusions}

Organoids have the capacity to mimic specific aspects of the 3D architecture, cell type composition and functionality of real organs while maintaining the advantages of simplified and easily accessible cell culture models. As such, they hold great promise for a range of 
biological and biomedical applications. In studies of basic tissue and organ biology, the potential for exploiting organoids to recapitulate in vivo phenomena has already been clearly revealed. Perhaps the most exciting perspective in basic organoid research is the ability to study human development (and disease) without tissue accessibility constraints. However, although the use of organoids also addresses some ethical issues, the prospect of generating in vitro human embryo-like structures is not free from ethical concerns ${ }^{164-166}$.

The path towards a broad-ranging translation of organoid technology into real-life preclinical and clinical applications is considerably more challenging. Nevertheless, as discussed above, studies are now emerging that demonstrate the potential of organoids in personalized medicine settings ${ }^{101,103}$, drug discovery ${ }^{99,105,106}$, regenerative medicine and gene therapy ${ }^{114}$, suggesting that more widespread adoption of organoids in these fields will become a reality in the coming decades.

Much has already been achieved in revealing the amazing level of self-organization that stem cells can display when cultured under the appropriate 3D conditions and in expanding the list of organoid types. Groundbreaking explorative studies for the application of various organoid types have also been published. However, many limitations still exist. Major challenges include regulating self-organization to generate organoids that develop deterministically, robustly and to physiologically relevant shapes and sizes; prolonging organoid lifespan to create mature, functional tissues that reach homeostasis; and recapitulating multi-factorial pathologies by incorporating additional key tissue compartments of native organs (such as the vasculature or immune system). Overcoming these challenges will require a multidisciplinary approach, and lessons from the field of bioengineering are likely to be particularly impactful.

\section{References}

1. Murry, C. E. \& Keller, G. Differentiation of embryonic stem cells to clinically relevant populations: lessons from embryonic development. Cell 132, 661-680 (2008).

2. Clevers, H. Modeling development and disease with organoids. Cell 165, 1586-1597 (2016).

3. Lancaster, M. A. \& Knoblich, J. A. Organogenesis in a dish: modeling development and disease using organoid technologies. Science 345, 1247125 (2014).

4. Sasai, Y. Cytosystems dynamics in self-organization of tissue architecture. Nature 493, 318326 (2013). This article is an inspiring review on self-organization.

5. Rheinwald, J. G. \& Green, H. Serial cultivation of strains of human epidermal keratinocytes: the formation of keratinizing colonies from single cells. Cell 6, 331-343 (1975).

6. Ehrmann, R. L. \& Gey, G. O. The growth of cells on a transparent gel of reconstituted rattail collagen. J. Natl Cancer Inst. 16, 1375-1403 (1956).

7. Orkin, R. W. et al. A murine tumor producing a matrix of basement membrane. J. Exp. Med. 145, 204-220 (1977).

8. Harrison, R. G., Greenman, M. J., Mall, F. P. \& Jackson, C. M. Observations of the living developing nerve fiber. Anat. Rec. 1, 116-128 (1907). 
9. Michalopoulos, G. \& Pitot, H. C. Primary culture of parenchymal liver cells on collagen membranes. Morphological and biochemical observations. Exp. Cell Res. 94, 70-78 (1975).

10. Barcellos-Hoff, M. H., Aggeler, J., Ram, T. G. \& Bissell, M. J. Functional differentiation and alveolar morphogenesis of primary mammary cultures on reconstituted basement membrane. Development 105, 223-235 (1989).

11. Petersen, O. W., Rønnov-Jessen, L., Howlett, A. R. \& Bissell, M. J. Interaction with basement membrane serves to rapidly distinguish growth and differentiation pattern of normal and malignant human breast epithelial cells. Proc. Natl Acad. Sci. USA 89, 9064-9068 (1992).

12. Eiraku, M. et al. Self-organizing optic-cup morphogenesis in three-dimensional culture. Nature 472, 51-56 (2011). This landmark study reports the capacity of PSCs to self-organize into the complex structure of an optic cup.

13. Sato, T. et al. Single Lgr5 stem cells build crypt-villus structures in vitro without a mesenchymal niche. Nature 459, 262-265 (2009). This landmark study reports the derivation of intestinal organoids from single adult ISCs.

14. Eiraku, M. et al. Self-organized formation of polarized cortical tissues from ESCs and its active manipulation by extrinsic signals. Cell Stem Cell 3, 519-532 (2008).nThis work highlights the self-organization properties of stem cells and describes the derivation of cerebral organoids from PSCs.

15. Fata, J. E. et al. The MAPKERK-1,2 pathway integrates distinct and antagonistic signals from TGF $\alpha$ and FGF7 in morphogenesis of mouse mammary epithelium. Dev. Biol. 306, 193207 (2007).

16. Guibert, C., Savineau, J. P., Crevel, H., Marthan, R. \& Rousseau, E. Effect of short-term organoid culture on the pharmaco-mechanical properties of rat extra- and intrapulmonary arteries. Br. J. Pharmacol. 146, 692-701 (2005).

17. Simian, M. et al. The interplay of matrix metalloproteinases, morphogens and growth factors is necessary for branching of mammary epithelial cells. Development 128, 3117-3131 (2001).

18. Zhang, Y. S. et al. Multisensor-integrated organs-on-chips platform for automated and continual in situ monitoring of organoid behaviors. Proc. Natl Acad. Sci. USA 114, E2293E2302 (2017).

19. Simian, M. \& Bissell, M. J. Organoids: a historical perspective of thinking in three dimensions. J. Cell Biol. 216, 31-40 (2016).

20. Langer, R. \& Vacanti, J. P. Tissue engineering. Science 260, 920-926 (1993).

21. Drost, J. et al. Organoid culture systems for prostate epithelial and cancer tissue. Nat. Protoc. 11, 347-358 (2016). 
22. Kelava, I. \& Lancaster, M. A. Dishing out mini-brains: current progress and future prospects in brain organoid research. Dev. Biol. 420, 199-209 (2016).

23. Kretzschmar, K. \& Clevers, H. Organoids: modeling development and the stem cell niche in a dish. Dev. Cell 38, 590-600 (2016).

24. Simunovic, M. \& Brivanlou, A. H. Embryoids, organoids and gastruloids: new approaches to understanding embryogenesis. Development 144, 976-985 (2017).

25. Takasato, M. \& Little, M. H. A strategy for generating kidney organoids: recapitulating the development in human pluripotent stem cells. Dev. Biol. 420, 210-220 (2016).

26. Yin, X. et al. Engineering stem cell organoids. Cell Stem Cell 18, 25-38 (2016).

27. Turner, D. A., Baillie-Johnson, P. \& Martinez Arias, A. Organoids and the genetically encoded self-assembly of embryonic stem cells. BioEssays 38, 181-191 (2016).

28. Green, J. B. A. \& Sharpe, J. Positional information and reaction-diffusion: two big ideas in developmental biology combine. Development 142, 1203-1211 (2015).

29. Turing, A. M. The chemical basis of morphogenesis. Phil. Trans. R. Soc. Lond. 237, 37-72 (1952).

30. Ferrell, J. E. Bistability, bifurcations, and Waddington's epigenetic landscape. Curr. Biol. 22, R458-R466 (2012).

31. Mori, H., Gjorevski, N., Inman, J. L., Bissell, M. J. \& Nelson, C. M. Self-organization of engineered epithelial tubules by differential cellular motility. Proc. Natl Acad. Sci. USA 106, 14890-14895 (2009).

32. Lancaster, M. A. et al. Cerebral organoids model human brain development and microcephaly. Nature 501, 373-379 (2013). This pioneering work reports the derivation of human cerebral organoids featuring coexisting, discrete brain regions. This work is also among the first to use an organoid to model a pathological condition.

33. McCracken, K. W. et al. Modelling human development and disease in pluripotent stemcell-derived gastric organoids. Nature 516, 400-404 (2014).

34. Linnemann, J. R. et al. Quantification of regenerative potential in primary human mammary epithelial cells. Development 142, 3239-3251 (2015).

35. Sachs, N., Tsukamoto, Y., Kujala, P., Peters, P. J. \& Clevers, H. Intestinal epithelial organoids fuse to form self-organizing tubes in floating collagen gels. Development 144, 1107-1112 (2017).

36. Gjorevski, N. et al. Designer matrices for intestinal stem cell and organoid culture. Nature 539, 560-564 (2016). 
37. Lindborg, B. A. et al. Rapid induction of cerebral organoids from human induced pluripotent stem cells using a chemically defined hydrogel and defined cell culture medium. Stem Cells Transl Med. 5, 970-979 (2016).

38. Muguruma, K., Nishiyama, A., Kawakami, H., Hashimoto, K. \& Sasai, Y. Self-organization of polarized cerebellar tissue in 3D culture of human pluripotent stem cells. Cell Rep. 10, 537550 (2015).

39. Sakaguchi, H. et al. Generation of functional hippocampal neurons from self-organizing human embryonic stem cell-derived dorsomedial telencephalic tissue. Nat. Commun. 6, 8896 (2015).

40. Takasato, M. et al. Directing human embryonic stem cell differentiation towards a renal lineage generates a self-organizing kidney. Nat. Cell Biol. 16, 118-126 (2014).

41. Takasato, M. et al. Kidney organoids from human iPS cells contain multiple lineages and model human nephrogenesis. Nature 536, 238 (2016).

42. McCracken, $\mathrm{K}$. W. et al. Wnt/ $\beta$-catenin promotes gastric fundus specification in mice and humans. Nature 541, 182-187 (2017).

43. Takebe, T. et al. Vascularized and functional human liver from an iPSC-derived organ bud transplant. Nature 499, 481-484 (2013). This work reports the formation of vascularized hPSC-derived liver organoids, demonstrating the possibility to obtain vascularized organoids by co-culturing stem cells and endothelial cells.

44. Stange, D. E. et al. Differentiated Troy+ chief cells act as reserve stem cells to generate all lineages of the stomach epithelium. Cell 155, 357-368 (2013).

45. Spence, J. R. et al. Directed differentiation of human pluripotent stem cells into intestinal tissue in vitro. Nature 470, 105-109 (2011).

46. Fordham, R. P. et al. Transplantation of expanded fetal intestinal progenitors contributes to colon regeneration after injury. Cell Stem Cell 13, 734-744 (2013).

47. Greggio, C. et al. Artificial three-dimensional niches deconstruct pancreas development in vitro. Development 140, 4452-4462 (2013).

48. Taguchi, A. et al. Redefining the in vivo origin of metanephric nephron progenitors enables generation of complex kidney structures from pluripotent stem cells. Cell Stem Cell 14, 5367 (2014). References 40 and 48 are among the first reports of organoids of mesodermal origin (kidney organoids).

49. Jamieson, P. R. et al. Derivation of a robust mouse mammary organoid system for studying tissue dynamics. Development 144, 1065-1071 (2017).

50. Maimets, M. et al. Long-term in vitro expansion of salivary gland stem cells driven by Wnt signals. Stem Cell Rep. 6, 150-162 (2016). 
51. Ren, W. et al. Single Lgr5- or Lgr6-expressing taste stem/progenitor cells generate taste bud cells ex vivo. Proc. Natl Acad. Sci. USA 111, 16401-16406 (2014).

52. Panciera, T. et al. Induction of expandable tissue-specific stem/progenitor cells through transient expression of YAP/TAZ. Cell Stem Cell 19, 725-737 (2016).

53. Sato, T. et al. Long-term expansion of epithelial organoids from human colon, adenoma, adenocarcinoma, and Barrett's epithelium. Gastroenterology 141, 1762-1772 (2011).

54. Barker, N. et al. Lgr5(+ve) stem cells drive self-renewal in the stomach and build long-lived gastric units in vitro. Cell Stem Cell 6, 25-36 (2010).

55. Bartfeld, S. et al. In vitro expansion of human gastric epithelial stem cells and their responses to bacterial infection. Gastroenterology 148, 126-136.e6 (2015).

56. Huch, M. et al. Unlimited in vitro expansion of adult bi-potent pancreas progenitors through the Lgr5/R-spondin axis. EMBO J. 32, 2708-2721 (2013).

57. Huch, M. et al. In vitro expansion of single Lgr5+ liver stem cells induced by Wnt-driven regeneration. Nature 494, 247-250 (2013).

58. Azzolin, L. et al. YAP/TAZ incorporation in the $\beta$-catenin destruction complex orchestrates the Wnt response. Cell 158, 157-170 (2014).

59. Xinaris, C., Brizi, V. \& Remuzzi, G. Organoid models and applications in biomedical research. Nephron 130, 191-199 (2015).

60. McCauley, H. A. \& Wells, J. M. Pluripotent stem cell-derived organoids: using principles of developmental biology to grow human tissues in a dish. Development 144, 958-962 (2017).

61. Rivron, N. C. et al. Blastocyst-like structures generated solely from stem cells. Nature 557, 106-111 (2018).

62. Harrison, S. E., Sozen, B., Christodoulou, N., Kyprianou, C. \& Zernicka-Goetz, M. Assembly of embryonic and extraembryonic stem cells to mimic embryogenesis in vitro. Science 356, eaal1810 (2017).

63. Shao, Y. et al. Self-organized amniogenesis by human pluripotent stem cells in a biomimetic implantation-like niche. Nat. Mater. 16, 419-425 (2017).

64. Shao, Y. et al. A pluripotent stem cell-based model for post-implantation human amniotic sac development. Nat. Commun. 8, 208 (2017).

65. Baillie-Johnson, P., van den Brink, S. C., Balayo, T., Turner, D. A. \& Martinez Arias, A. Generation of aggregates of mouse embryonic stem cells that show symmetry breaking, polarization and emergent collective behaviour in vitro. J. Vis. Exp. 105, e53252 (2015).

66. van den Brink, S. C. et al. Symmetry breaking, germ layer specification and axial organisation in aggregates of mouse embryonic stem cells. Development 141, 4231-4242 
(2014). This work shows the capacity of mouse PSCs to recapitulate, in 3D, complex gastrulation-like processes that resemble those occurring in the early embryo.

67. Etoc, F. et al. A balance between secreted inhibitors and edge sensing controls gastruloid self-organization. Dev. Cell 39, 302-315 (2016).

68. Martyn, I., Kanno, T. Y., Ruzo, A., Siggia, E. D. \& Brivanlou, A. H. Self-organization of a human organizer by combined Wnt and Nodal signalling. Nature 558, 132-135 (2018).

69. Morgani, S. M., Metzger, J. J., Nichols, J., Siggia, E. D. \& Hadjantonakis, A. K. Micropattern differentiation of mouse pluripotent stem cells recapitulates embryo regionalized cell fate patterning. elife 7, e32839 (2018).

70. Tewary, M. et al. A stepwise model of reaction-diffusion and positional-information governs self-organized human peri-gastrulation-like patterning. Development 144, 42984312 (2017).

71. Warmflash, A., Sorre, B., Etoc, F., Siggia, E. D. \& Brivanlou, A. H. A method to recapitulate early embryonic spatial patterning in human embryonic stem cells. Nat. Methods 11, 847854 (2014). This work highlights the capacity of human ESCs to self-organize into patterned germ layers.

72. Meinhardt, A. et al. 3D reconstitution of the patterned neural tube from embryonic stem cells. Stem Cell Rep. 3, 987-999 (2014).

73. Ranga, A. et al. Neural tube morphogenesis in synthetic 3D microenvironments. Proc. Natl Acad. Sci. USA 113, E6831-E6839 (2016).

74. Sato, T. et al. Paneth cells constitute the niche for Lgr5 stem cells in intestinal crypts. Nature 469, 415-418 (2011).

75. Sato, T. \& Clevers, H. Growing self-organizing mini-guts from a single intestinal stem cell: mechanism and applications. Science 340, 1190-1194 (2013).

76. Farin, H. F. et al. Visualization of a short-range Wnt gradient in the intestinal stem-cell niche. Nature 530, 340-343 (2016).

77. Snippert, H. J. et al. Intestinal crypt homeostasis results from neutral competition between symmetrically dividing Lgr5 stem cells. Cell 143, 134-144 (2010).

78. Nakano, T. et al. Self-formation of optic cups and storable stratified neural retina from human ESCs. Cell Stem Cell 10, 771-785 (2012).

79. Dekkers, J. F. et al. A functional CFTR assay using primary cystic fibrosis intestinal organoids. Nat. Med. 19, 939-945 (2013). This article provides one of the first examples of the development of an organoid-based functional assay for drug discovery and/or screening.

80. Huch, M. et al. Long-term culture of genome-stable bipotent stem cells from adult human liver. Cell 160, 299-312 (2015). 
81. Xia, Y. et al. Directed differentiation of human pluripotent cells to ureteric bud kidney progenitor-like cells. Nat. Cell Biol. 15, 1507-1515 (2013).

82. Castellanos-Gonzalez, A., Cabada, M. M., Nichols, J., Gomez, G. \& White, A. C. Human primary intestinal epithelial cells as an improved in vitro model for Cryptosporidium parvum infection. Infect. Immun. 81, 1996-2001 (2013).

83. Cugola, F. R. et al. The Brazilian Zika virus strain causes birth defects in experimental models. Nature 534, 267-271 (2016).

84. Dang, J. et al. Zika virus depletes neural progenitors in human cerebral organoids through activation of the innate immune receptor TLR3. Cell Stem Cell 19, 258-265 (2016).

85. Farin, H. F. et al. Paneth cell extrusion and release of antimicrobial products is directly controlled by immune cell-derived IFN- $\gamma$. J. Exp. Med. 211, 1393-1405 (2014).

86. Finkbeiner, S. R. et al. Stem cell-derived human intestinal organoids as an infection model for rotaviruses. mBio 3, e00159-12 (2012).

87. Forbester, J. L. et al. Interaction of Salmonella enterica serovar typhimurium with intestinal organoids derived from human induced pluripotent stem cells. Infect. Immun. 83, 2926-2934 (2015).

88. Garcez, P. P. et al. Zika virus impairs growth in human neurospheres and brain organoids. Science 352, 816-818 (2016).

89. Leslie, J. L. et al. Persistence and toxin production by Clostridium difficile within human intestinal organoids result in disruption of epithelial paracellular barrier function. Infect. Immun. 83, 138-145 (2015).

90. Qian, X. et al. Brain-region-specific organoids using mini-bioreactors for modeling ZIKV exposure. Cell 165, 1238-1254 (2016).

91. Tao, L. et al. Frizzled proteins are colonic epithelial receptors for C. difficile toxin B. Nature 538, 350-355 (2016).

92. Wilson, S. S., Tocchi, A., Holly, M. K., Parks, W. C. \& Smith, J. G. A small intestinal organoid model of non-invasive enteric pathogen-epithelial cell interactions. Mucosal Immunol. 8, 352-361 (2014).

93. Zhang, Y. G., Wu, S., Xia, Y. \& Sun, J. Salmonella-infected crypt-derived intestinal organoid culture system for host-bacterial interactions. Physiol. Rep. 2, e12147 (2014).

94. Zomer-van Ommen, D. D. et al. Functional characterization of cholera toxin inhibitors using human intestinal organoids. J. Med. Chem. 59, 6968-6972 (2016).

95. Boj, S. F. et al. Organoid models of human and mouse ductal pancreatic cancer. Cell 160, 324-338 (2015). 
96. Gao, D. et al. Organoid cultures derived from patients with advanced prostate cancer. Cell 159, 176-187 (2014).

97. Hubert, C. G. et al. A three-dimensional organoid culture system derived from human glioblastomas recapitulates the hypoxic gradients and cancer stem cell heterogeneity of tumors found in vivo. Cancer Res. 76, 2465-2477 (2016).

98. Yeung, T. M., Gandhi, S. C., Wilding, J. L., Muschel, R. \& Bodmer, W. F. Cancer stem cells from colorectal cancer-derived cell lines. Proc. Natl Acad. Sci. USA 107, 3722-3727 (2010).

99. Zhou, T. et al. High-content screening in hPSC-neural progenitors identifies drug candidates that inhibit zika virus infection in fetal-like organoids and adult brain. Cell Stem Cell 21, 274-283 (2017).

100. Davies, J. C., Alton, E. W. F. W. \& Bush, A. Cystic fibrosis. BMJ 335, 1255-1259 (2007).

101. Saini, A. Cystic fibrosis patients benefit from mini guts. Cell Stem Cell 19, 425-427 (2016).

102. Czerniecki, S. M. et al. High-throughput screening enhances kidney organoid differentiation from human pluripotent stem cells and enables automated multidimensional phenotyping. Cell Stem Cell 22, 929-940.e4 (2018).

103. Dekkers, J. F. et al. Characterizing responses to CFTR-modulating drugs using rectal organoids derived from subjects with cystic fibrosis. Sci. Transl Med. 8, 344ra84 (2016).

104. Fujii, M. et al. A colorectal tumor organoid library demonstrates progressive loss of niche factor requirements during tumorigenesis. Cell Stem Cell 18, 827-838 (2016).

105. van de Wetering, $M$. et al. Prospective derivation of a living organoid biobank of colorectal cancer patients. Cell 161, 933-945 (2015).

106. Vlachogiannis, G. et al. Patient-derived organoids model treatment response of metastatic gastrointestinal cancers. Science 359, 920-926 (2018).

107. Lee, S. H. et al. Tumor evolution and drug response in patient-derived organoid models of bladder cancer. Cell 173, 515-528.e17 (2018).

108. Mariani, J. et al. FOXG1-dependent dysregulation of GABA/glutamate neuron differentiation in autism spectrum disorders. Cell 162, 375-390 (2015).

109. Tannenbaum, J. \& Bennett, B. T. Russell and Burch's 3Rs then and now: the need for clarity in definition and purpose. J. Am. Assoc. Lab. Anim. Sci. 54, 120-132 (2015).

110. Assawachananont, J. et al. Transplantation of embryonic and induced pluripotent stem cell-derived 3D retinal sheets into retinal degenerative mice. Stem Cell Rep. 2, 662-674 (2014).

111. Mandai, M. et al. iPSC-derived retina transplants improve vision in rd1 end-stage retinaldegeneration mice. Stem Cell Rep. 8, 69-83 (2017). 
112. Shirai, H. et al. Transplantation of human embryonic stem cell-derived retinal tissue in two primate models of retinal degeneration. Proc. Natl Acad. Sci. USA 113, E81-E90 (2016).

113. Yui, S. et al. Functional engraftment of colon epithelium expanded in vitro from a single adult Lgr5+ stem cell. Nat. Med 18, 618-623 (2012).

114. Schwank, G. et al. Functional repair of CFTR by CRISPR/Cas9 in intestinal stem cell organoids of cystic fibrosis patients. Cell Stem Cell 13, 653-658 (2013).

115. Camp, J. G. et al. Human cerebral organoids recapitulate gene expression programs of fetal neocortex development. Proc. Natl Acad. Sci. USA 112, 15672-15677 (2015).

116. Quadrato, G. et al. Cell diversity and network dynamics in photosensitive human brain organoids. Nature 545, 48-53 (2017).

117. Camp, J. G. et al. Multilineage communication regulates human liver bud development from pluripotency. Nature 546, 533-538 (2017).

118. Scialdone, A. et al. Resolving early mesoderm diversification through single-cell expression profiling. Nature 535, 289-293 (2016).

119. Junker, J. P. et al. Genome-wide RNA tomography in the zebrafish embryo. Cell 159, 662675 (2014).

120. Grubb, M. S. \& Thompson, I. D. The influence of early experience on the development of sensory systems. Curr. Opin. Neurobiol. 14, 503-512 (2004).

121. Martin, I., Wendt, D. \& Heberer, M. The role of bioreactors in tissue engineering. Trends Biotechnol. 22, 80-86 (2004).

122. Zhao, J. et al. Bioreactors for tissue engineering: an update. Biochem. Eng. J. 109, 268281 (2016).

123. Lancaster, M. A. \& Knoblich, J. A. Generation of cerebral organoids from human pluripotent stem cells. Nat. Protoc. 9, 2329-2340 (2014).

124. Miller, J. S. et al. Rapid casting of patterned vascular networks for perfusable engineered three-dimensional tissues. Nat. Mater. 11, 768-774 (2012).

125. Tocchio, A. et al. Versatile fabrication of vascularizable scaffolds for large tissue engineering in bioreactor. Biomaterials 45, 124-131 (2015).

126. Wang, X. Y. et al. Engineering interconnected 3D vascular networks in hydrogels using molded sodium alginate lattice as the sacrificial template. Lab. Chip 14, 2709-2716 (2014).

127. Brandenberg, N. \& Lutolf, M. P. In situ patterning of microfluidic networks in 3D cellladen hydrogels. Adv. Mater. 28, 7450-7456 (2016).

128. Zhang, Y. S. et al. Bioprinting 3D microfibrous scaffolds for engineering endothelialized myocardium and heart-on-a-chip. Biomaterials 110, 45-59 (2016). 
129. Zhu, W. et al. Direct 3D bioprinting of prevascularized tissue constructs with complex microarchitecture. Biomaterials 124, 106-115 (2017).

130. Pham, M. T. et al. Generation of human vascularized brain organoids. Neuroreport 29, 588-593 (2018).

131. Mansour, A. A. et al. An in vivo model of functional and vascularized human brain organoids. Nat. Biotechnol 36, 432-441 (2018).

132. Watson, C. L. et al. An in vivo model of human small intestine using pluripotent stem cells. Nat. Med. 20, 1310-1314 (2014).

133. Dye, B. R. et al. A bioengineered niche promotes in vivo engraftment and maturation of pluripotent stem cell derived human lung organoids. eLife 5, e19732 (2016).

134. Miller, J. D. et al. Human iPSC-based modeling of late-onset disease via progerin-induced aging. Cell Stem Cell 13, 691-705 (2013).

135. Wang, Y. et al. Formation of human colonic crypt array by application of chemical gradients across a shaped epithelial monolayer. Cell. Mol. Gastroenterol. Hepatol. 5, 113-130 (2018).

136. Wang, Y. et al. A microengineered collagen scaffold for generating a polarized crypt-villus architecture of human small intestinal epithelium. Biomaterials 128, 44-55 (2017).

137. Ehrbar, M. et al. Biomolecular hydrogels formed and degraded via site-specific enzymatic reactions. Biomacromolecules 8, 3000-3007 (2007).

138. Kim, S., Chung, E. H., Gilbert, M. \& Healy, K. E. Synthetic MMP-13 degradable ECMs based on poly( $\mathrm{N}$-isopropylacrylamide-co-acrylic acid) semi-interpenetrating polymer networks. I. Degradation and cell migration. J. Biomed. Mater. Res. A 75, 73-88 (2005).

139. Kloxin, A. M., Kasko, A. M., Salinas, C. N. \& Anseth, K. S. Photodegradable hydrogels for dynamic tuning of physical and chemical properties. Science 324, 59-63 (2009).

140. Lutolf, M. P. \& Hubbell, J. A. Synthesis and physicochemical characterization of endlinked poly(ethylene glycol)-co-peptide hydrogels formed by Michael-type addition. Biomacromolecules 4, 713-722 (2003).

141. Mann, B. K., Gobin, A. S., Tsai, A. T., Schmedlen, R. H. \& West, J. L. Smooth muscle cell growth in photopolymerized hydrogels with cell adhesive and proteolytically degradable domains: synthetic ECM analogs for tissue engineering. Biomaterials 22, 3045-3051 (2001).

142. Peyton, S. R., Raub, C. B., Keschrumrus, V. P. \& Putnam, A. J. The use of poly(ethylene glycol) hydrogels to investigate the impact of ECM chemistry and mechanics on smooth muscle cells. Biomaterials 27, 4881-4893 (2006).

143. Phelps, E. A. et al. Maleimide cross-linked bioactive PEG hydrogel exhibits improved reaction kinetics and cross-linking for cell encapsulation and in situ delivery. Adv. Mater. 24, 64-70 (2012). 
144. Tsurkan, M. V. et al. Defined polymer-peptide conjugates to form cell-instructive starPEG-heparin matrices in situ. Adv. Mater. 25, 2606-2610 (2013).

145. Wylie, R. G. et al. Spatially controlled simultaneous patterning of multiple growth factors in three-dimensional hydrogels. Nat. Mater. 10, 799-806 (2011).

146. Cruz-Acuña, R. et al. Synthetic hydrogels for human intestinal organoid generation and colonic wound repair. Nat. Cell Biol. 19, 1326-1335 (2017).

147. DeForest, C. A., Polizzotti, B. D. \& Anseth, K. S. Sequential click reactions for synthesizing and patterning three-dimensional cell microenvironments. Nat. Mater. 8, 659-664 (2009).

148. Mosiewicz, K. A. et al. In situ cell manipulation through enzymatic hydrogel photopatterning. Nat. Mater. 12, 1072-1078 (2013).

149. Dankers, P. Y., Harmsen, M. C., Brouwer, L. A., van Luyn, M. J. \& Meijer, E. W. A modular and supramolecular approach to bioactive scaffolds for tissue engineering. Nat. Mater. 4, 568-574 (2005).

150. Gelain, F., Bottai, D., Vescovi, A. \& Zhang, S. Designer self-assembling peptide nanofiber scaffolds for adult mouse neural stem cell 3-dimensional cultures. PLOS ONE 1, e119 (2006).

151. Silva, G. A. et al. Selective differentiation of neural progenitor cells by high-epitope density nanofibers. Science 303, 1352-1355 (2004).

152. Bajaj, P., Schweller, R. M., Khademhosseini, A., West, J. L. \& Bashir, R. 3D biofabrication strategies for tissue engineering and regenerative medicine. Annu. Rev. Biomed. Eng. 16, 247-276 (2014).

153. Todhunter, M. E. et al. Programmed synthesis of three-dimensional tissues. Nat. Methods 12, 975-981 (2015).

154. Hughes, A. J. et al. Engineered tissue folding by mechanical compaction of the mesenchyme. Dev. Cell 44, 165-178. e6 (2018).

155. Demers, C. J. et al. Development-on-chip: in vitro neural tube patterning with a microfluidic device. Development 143, 1884-1892 (2016).

156. Keenan, T. M. \& Folch, A. Biomolecular gradients in cell culture systems. Lab. Chip 8, 3457 (2008).

157. Tabata, Y. \& Lutolf, M. P. Multiscale microenvironmental perturbation of pluripotent stem cell fate and self-organization. Sci. Rep. 7, 44711 (2017).

158. Carpenedo, R. L. et al. Homogeneous and organized differentiation within embryoid bodies induced by microsphere-mediated delivery of small molecules. Biomaterials 30, 25072515 (2009).

159. Morsut, L. et al. Engineering customized cell sensing and response behaviors using synthetic notch receptors. Cell 164, 780-791 (2016). 
160. Toda, S., Blauch, L. R., Tang, S. K. Y., Morsut, L. \& Lim, W. A. Programming self-organizing multicellular structures with synthetic cell-cell signaling. Science 361, 156-162 (2018).

161. Workman, M. J. et al. Engineered human pluripotent-stem-cell-derived intestinal tissues with a functional enteric nervous system. Nat. Med. 23, 49-59 (2017).

162. Zhang, C., Zhao, Z., Abdul Rahim, N. A., van Noort, D. \& Yu, H. Towards a human-on-chip: culturing multiple cell types on a chip with compartmentalized microenvironments. Lab. Chip 9, 3185-3192 (2009).

163. Zhang, L. et al. Establishing estrogen-responsive mouse mammary organoids from single Lgr5+ cells. Cell. Signal. 29, 41-51 (2017).

164. Pera, M. F. et al. What if stem cells turn into embryos in a dish? Nat. Methods 12,917 (2015).

165. Shen, H. Embryo assembly 101. Nature 559, 19-22 (2018).

166. Hyun, I. Engineering ethics and self-organizing models of human development: opportunities and challenges. Cell Stem Cell 21, 718-720 (2017).

167. Kuwahara, A. et al. Generation of a ciliary margin-like stem cell niche from self-organizing human retinal tissue. Nat. Commun. 6, 6286 (2015).

168. Wahlin, K. J. et al. Photoreceptor outer segment-like structures in long-term 3D retinas from human pluripotent stem cells. Sci. Rep. 7, 766 (2017).

169. Zhong, X. et al. Generation of three-dimensional retinal tissue with functional photoreceptors from human iPSCs. Nat. Commun. 5, 4047 (2014).

170. Otani, T., Marchetto, M. C., Gage, F. H., Simons, B. D. \& Livesey, F. J. 2D and 3D stem cell models of primate cortical development identify species-specific differences in progenitor behavior contributing to brain size. Cell Stem Cell 18, 467-480 (2016).

171. Lancaster, M. A. et al. Guided self-organization and cortical plate formation in human brain organoids. Nat. Biotechnol. 35, 659-666 (2017).

172. Suga, H. et al. Self-formation of functional adenohypophysis in three-dimensional culture. Nature 480, 57-62 (2011).

173. Noguchi, T. K. et al. Generation of stomach tissue from mouse embryonic stem cells. Nat. Cell Biol. 17, 984-993 (2015).

174. Fukuda, M. et al. Small intestinal stem cell identity is maintained with functional Paneth cells in heterotopically grafted epithelium onto the colon. Genes Dev. 28, 1752-1757 (2014).

175. von Furstenberg, R. J. et al. Sorting mouse jejunal epithelial cells with CD24 yields a population with characteristics of intestinal stem cells. Am. J. Physiol. Gastrointest. Liver Physiol. 300, G409-G417 (2011). 
176. Gracz, A. D., Ramalingam, S. \& Magness, S. T. Sox9 expression marks a subset of CD24expressing small intestine epithelial stem cells that form organoids in vitro. Am. J. Physiol. Gastrointest. Liver Physiol. 298, G590-G600 (2010).

177. Hannan, N. R. F. et al. Generation of multipotent foregut stem cells from human pluripotent stem cells. Stem Cell Rep. 1, 293-306 (2013).

178. Ootani, A. et al. Sustained in vitro intestinal epithelial culture within a Wnt-dependent stem cell niche. Nat. Med 15, 701-706 (2009).

179. Jung, P. et al. Isolation and in vitro expansion of human colonic stem cells. Nat. Med 17, 1225-1227 (2011).

180. Broutier, L. et al. Culture and establishment of self-renewing human and mouse adult liver and pancreas 3D organoids and their genetic manipulation. Nat. Protoc. 11, 1724-1743 (2016).

181. Rock, J. R. et al. Basal cells as stem cells of the mouse trachea and human airway epithelium. Proc. Natl Acad. Sci. USA 106, 12771-12775 (2009).

182. Tadokoro, T. et al. IL-6/STAT3 promotes regeneration of airway ciliated cells from basal stem cells. Proc. Natl Acad. Sci. USA 111, E3641-E3649 (2014).

183. Wong, A. P. et al. Directed differentiation of human pluripotent stem cells into mature airway epithelia expressing functional CFTR protein. Nat. Biotechnol. 30, 876-882 (2012).

184. Huang, S. X. L. et al. Highly efficient generation of airway and lung epithelial cells from human pluripotent stem cells. Nat. Biotechnol. 32, 84-91 (2014).

185. Jain, R. et al. Plasticity of Hopx(+) type I alveolar cells to regenerate type II cells in the lung. Nat. Commun. 6, 6727 (2015).

186. Barkauskas, C. E. et al. Type 2 alveolar cells are stem cells in adult lung. J. Clin. Invest. 123, 3025-3036 (2013).

187. Dye, B. R. et al. In vitro generation of human pluripotent stem cell derived lung organoids. elife 4, e05098 (2015).

188. Kurmann, A. A. et al. Regeneration of thyroid function by transplantation of differentiated pluripotent stem cells. Cell Stem Cell 17, 527-542 (2015).

189. DeWard, A. D., Cramer, J. \& Lagasse, E. Cellular heterogeneity in the mouse esophagus implicates the presence of a nonquiescent epithelial stem cell population. Cell Rep. 9, 701711 (2014).

190. Chua, C. W. et al. Single luminal epithelial progenitors can generate prostate organoids in culture. Nat. Cell Biol. 16, 951-961 (2014).

191. Karthaus, W. R. et al. Identification of multipotent luminal progenitor cells in human prostate organoid cultures. Cell 159, 163-175 (2014). 
192. Kessler, M. et al. The Notch and Wnt pathways regulate stemness and differentiation in human fallopian tube organoids. Nat. Commun. 6, 8989 (2015).

193. Morizane, R. et al. Nephron organoids derived from human pluripotent stem cells model kidney development and injury. Nat. Biotechnol. 33, 1193-1200 (2015).

194. Freedman, B. S. et al. Modelling kidney disease with CRISPR-mutant kidney organoids derived from human pluripotent epiblast spheroids. Nat. Commun. 6, 8715 (2015).

195. Sachs, N. et al. A living biobank of breast cancer organoids captures disease heterogeneity. Cell 172, 373-386.e10 (2018).

196. Jardé, T. et al. Wnt and Neuregulin1/ErbB signaling extends 3D culture of hormone responsive mammary organoids. Nat. Commun. 7, 13207 (2016).

197. Lombaert, I. M. A. et al. Rescue of salivary gland function after stem cell transplantation in irradiated glands. PLOS ONE 3, e2063 (2008).

198. Nanduri, L. S. Y. et al. Purification and ex vivo expansion of fully functional salivary gland stem cells. Stem Cell Rep. 3, 957-964 (2014).

199. Feng, J., van der Zwaag, M., Stokman, M. A., van Os, R. \& Coppes, R. P. Isolation and characterization of human salivary gland cells for stem cell transplantation to reduce radiation-induced hyposalivation. Radiother. Oncol. 92, 466-471 (2009). 
Table1

\begin{tabular}{|c|c|c|c|}
\hline TISSUE or ORGAN & SOURCE & TRANSLATIONALAPPLCATIONS & $\begin{array}{l}\text { TECHNOLOGICAL } \\
\text { IMPLEMENTATION }\end{array}$ \\
\hline \multicolumn{4}{|l|}{ Neuroectoderm } \\
\hline $\begin{array}{l}\text { OPTICCUPAND } \\
\text { RETINA }\end{array}$ & $\begin{array}{c}\text { MOUSE PSCS } \\
\text { HUMAN PSCS }{ }^{12,112,110,67-1 \oplus}\end{array}$ & $\begin{array}{l}\text {-Orthotopic transplantation of mouse retinal organoids in murine models } \\
\text { of retinal degeneration }{ }^{110} \\
\text {-Orthotopic transplantation of human retinal organdids in primate modss } \\
\text { of retinal degeneration }{ }^{112}\end{array}$ & \\
\hline $\begin{array}{c}\text { CEREBRAL } \\
\text { STRUCTURES } \\
\text { (CEREBRAL } \\
\text { NEOCORTEX, } \\
\text { OLFACTORY BULB, } \\
\text { HIPPOCAMPUS, } \\
\text { HYPOTHALAMUS, } \\
\text { MIDBRAIN } \\
\text { STRUCTURES, } \\
\text { CHOROID PLEXUS) }\end{array}$ & $\begin{array}{c}\text { MOUSE PSCS }{ }^{14,32} \\
\text { PRIMATE PSCS }{ }^{170} \\
\text { HUMAN PSCS }{ }^{14,32,3990,99171}\end{array}$ & $\begin{array}{l}\text {-Model of microcephalyfrompatient-derived hipSC cerebrad organoids }{ }^{32} \\
\text {-Model of Zika virus infection on forebrain organoids }{ }^{90} \\
\text {-Drug screening for Zikavirusinfection using forebrain orgamids as } \\
\text { models } 5^{99}\end{array}$ & $\begin{array}{l}\text {-Use of standard bioreactors } \\
\text { to obtain human cerebral } \\
\text { organoids with extended } \\
\text { lifespan }{ }^{32} \\
\text {-Use of miniaturized spinning } \\
\text { bioreactors (Spiz-Omega)to } \\
\text { obtain human forebrain, } \\
\text { midbrain and hypothalamic } \\
\text { organoids in m multiplexed } \\
\text { fashion }{ }^{00} \\
\text {-Use of a chemicallly-defined } \\
\text { PLGAcopolymer as sc fffdd to } \\
\text { obtain elongated cerebral } \\
\text { organoids } 711\end{array}$ \\
\hline ADENOHYPOPHYSIS & MOUSE PSCS $^{172}$ & -Ectopic transplantation in hypphysectomized mice ${ }^{172}$ & - \\
\hline CEREBELLUM & HUMAN PSC ${ }^{38}$ & & \\
\hline
\end{tabular}




\begin{tabular}{|c|c|c|c|}
\hline \multicolumn{4}{|l|}{ Endoderm } \\
\hline GASTRICFUNDUS & HUMAN PSCs ${ }^{42}$ & & \\
\hline GASTRICCORPUS & $\begin{array}{c}\text { MOUSE PSCS }{ }^{173} \\
\text { MOUSE ASCS }{ }^{44} \\
\text { MOUSE dissociated } \\
\text { tissue }^{44,55} \\
\text { HUMAN dissociated tissue }\end{array}$ & $\begin{array}{c}\text {-Human gastric organoids originatedfrom } \\
\text { tumour cells to model human gastric } \\
\text { cancer }{ }^{55} \\
\text {-Model of Helic obacter pyloriinfection in } \\
\text { human gastric organoids to study } \\
\text { pathogenetic mechanisms } 5^{55}\end{array}$ & - \\
\hline $\begin{array}{l}\text { GASTRICPYLORIC } \\
\text { ANTRUM }\end{array}$ & $\begin{array}{l}\text { MOUSE PSCS }{ }^{173} \\
\text { MOUSE ASCS }{ }^{54} \\
\text { HUMAN PSCs }{ }^{33,42}\end{array}$ & $\begin{array}{l}\text {-Model of Helic obacter pyloriinfection in } \\
\text { human gastric organoids to study } \\
\text { pathogenetic mechanisms }\end{array}$ & \\
\hline SMALL INTESTINE & $\begin{array}{l}\text { MOUSE ASCS }{ }^{13,36,174176} \\
\text { HUMAN PSCS }{ }^{45,46,16,177} \\
\text { MOUSE dissociated tissue } \\
\text { HUMAN dissociated } \\
\text { tissue } 36,114,136 \\
\text { MOUSE dissociated foetal } \\
\text { tissue }\end{array}$ & $\begin{array}{l}\text {-Ectopic transplantation ofmouse small } \\
\text { intestinal organoids onto damaged mause } \\
\text { colonic epithelium }{ }^{174} \\
\text {-hPSC-derived intestinal organoids to } \\
\text { model congenital loss of intestinal } \\
\text { enteroendocrine cells } \\
\\
\text {-CRISPR-Cas9-mediated correction of CFIR } \\
\text { in intestinal organoids derived from cystic } \\
\text { fibrosis patients. Development ofan in } \\
\text { vitro readout to evaluate therecoveryof } \\
\text { CFTR function }\end{array}$ & $\begin{array}{l}\text {-Use of a synthetic chemic ally-defined hydrogel with tunable } \\
\text { mechanic al properties to support mouse and human } \\
\text { intestinal organoid culture } \\
\text {-Micro-structured collagen scaffolds mimic king thesmall } \\
\text { intestinal crypt-villus macro-architecture have been used to } \\
\text { culture human intestinal epithelium starting from intestinal } \\
\text { stem cells }{ }^{136}\end{array}$ \\
\hline $\begin{array}{c}\text { COLON and/or } \\
\text { LARGE INTESTINE }\end{array}$ & $\begin{array}{l}\text { MOUSE ASCS }{ }^{113} \\
\text { HUMAN PSCS }{ }^{45,46,16,177} \\
\text { HUMAN ASCS }{ }^{179} \\
\text { MOUSE dissociated } \\
\text { tissue } \\
\text { HUMAN dissociated } \\
\text { tissue }{ }^{33,114,135} \\
\\
\text { MOUSE dissociated foeta } \\
\text { tissue }^{46}\end{array}$ & $\begin{array}{l}\text {-Orthotopic transplantation of mouse } \\
\text { colon organoids to damaged mouse cdoric } \\
\text { epithelium }{ }^{113} \\
\text {-hPSC-derived intestinal organoids to } \\
\text { model congenital loss of intestinal } \\
\text { enteroendocrine cells } \\
\text { (5) } \\
\text {-Human colon organoids obtained from } \\
\text { tumour cells as models for human } \\
\text { colorectal cance }{ }^{53} \\
\text {-CRISPR-Cas9-mediated correction of CFIR } \\
\text { in intestinal organoids derived from cystic } \\
\text { fibrosis patients. Development ofan in } \\
\text { vitro readout to evaluate therec overy of } \\
\text { CFTR function }{ }^{114} \\
\text {-Transplantation of mouse intestinal } \\
\text { progenitor organoids on damaged colonic } \\
\text { epithelium }\end{array}$ & $\begin{array}{l}\text { Use of a synthetic chemic ally-defined hydrogel to grow } \mathrm{hPSC} \text { - } \\
\text { derived intestinal organoids in vitro and for their orthotopic } \\
\text { transplantation on damaged mouse colonic epithelium } \\
\text {-Micro-structured collagen scaffolds resem bling colonic } \\
\text { crypts array macro-architecture have been used to culture } \\
\text { self-renewing human colonic epithelium }\end{array}$ \\
\hline LIVER & $\begin{array}{l}\text { MOUSE ASCS } \\
\text { HUMAN PSCS } \\
\text { HUMAN ASCS } \\
\text { Ho }\end{array}$ & $\begin{array}{l}\text {-Transplantation of mouse liver organoids } \\
\text { into mouse model of type I tyrosinemia }{ }^{57} \\
\text {-Use of patient-derived liver organoids to } \\
\text { model alpha1-antitrypsin deficiencyand } \\
\text { Alagille syndrome }{ }^{80} \\
\end{array}$ & $\begin{array}{c}\text { Co-culture of hPSC-derived hepatic endodermal cells with } \\
\text { human endothelial (HUVEC) and human mesenchymal stem } \\
\text { cells allows for the formation of a more completeliver } \\
\text { cytoarchitecture }{ }^{43}\end{array}$ \\
\hline PANCREAS & $\begin{array}{l}\text { MOUSE ASCS } \\
\text { MOUSE dissociated tissue } \\
\text { HUMAN dissociated } \\
\text { tissue } \\
\begin{array}{l}95,180 \\
\text { MOUSE dissociated } \\
\text { embryonic tissue }\end{array}\end{array}$ & $\begin{array}{l}\text { Comparison between mouse pancreatic } \\
\text { organoids generated fromnormal, early } \\
\text { neoplastic and tumoural cells have } \\
\text { revealed new insights on genes involved in } \\
\text { the progression of pancreatic ductal } \\
\text { adenocarcinoma } a^{95}\end{array}$ & \\
\hline $\begin{array}{l}\text { TRACHEA/BRONCHI } \\
\text { (or UPPER AI RWAYS) }\end{array}$ & $\begin{array}{l}\text { MOUSE ASCS }{ }^{181,182} \\
\text { HUMAN PSCs } \\
\text { HUMA3,184 } \\
\text { HUSCs }{ }^{181}\end{array}$ & $\begin{array}{c}\text { Proof-of-principle experiment on patient } \\
\text { hPSC-derived epithelial airways for cystic } \\
\text { fibrosis drug screening }\end{array}$ & - \\
\hline
\end{tabular}




\begin{tabular}{|c|c|c|c|}
\hline $\begin{array}{l}\text { PULMONARY } \\
\text { ALVEOLUS }\end{array}$ & $\begin{array}{c}\text { MOUSE ASCs }{ }^{185,186} \\
\text { HUMAN PSCs }{ }^{133,184,187} \\
\text { HUMAN ASCS }{ }^{186} \\
\end{array}$ & - & - \\
\hline THYROID & MOUSE PSCS ${ }^{188}$ & - & - \\
\hline ESOPHAGUS & MOUSE dissociated tissue ${ }^{\mathbb{x}}$ & - & $=$ \\
\hline PROSTATE & $\begin{array}{c}\text { MOUSE ASCs }{ }^{190,191} \\
\text { HUMAN ASCs }{ }^{190,191} \\
\text { MOUSE dissociated tissue }\end{array}$ & $\begin{array}{l}\text { Genetically engineered mouse prostate } \\
\text { organoids have been used to model } \\
\text { prostate cancer }{ }^{190191}\end{array}$ & - \\
\hline FALLOPIAN TUBE & HUMAN ASCs ${ }^{192}$ & & \\
\hline
\end{tabular}

\begin{tabular}{|c|c|c|c|}
\hline \multicolumn{4}{|l|}{ Mesoderm } \\
\hline KIDNEY & $\begin{array}{c}\text { MOUSE PSCS } \\
\text { HUMAN PSCS } \\
\text { Ho,41,48193, } 14\end{array}$ & $\begin{array}{l}\text {-Use of human kidney organoids for the } \\
\text { evaluation of nephrotoxicity }{ }^{11,33,194} \\
\text {-Use of CRISPR-Cas } 9 \text { engineered human } \\
\text { kidney organoids to model polycystic } \\
\text { kidney disease }{ }^{194}\end{array}$ & - \\
\hline \multicolumn{4}{|l|}{ Surface Ectoderm } \\
\hline MAMMARY GLAND & $\begin{array}{l}\text { MOUSE ASCs }{ }^{49,163} \\
\text { HUMAN ASCs } s^{34,195} \\
\text { MOUSE dissociated } \\
\text { tissue }^{15,196}\end{array}$ & $\begin{array}{c}\text { Generation of a breast cancerorganoid } \\
\text { biobank }^{195}\end{array}$ & - \\
\hline TASTE BUD & MOUSE ASCS ${ }^{51}$ & - & - \\
\hline SALIVARY GLAND & $\begin{array}{l}\text { MOUSE ASCS } S^{50,197,19} \\
\text { HUMAN ASCs }{ }^{199}\end{array}$ & $\begin{array}{l}\text { Mouse salivary gland organoids havebeen } \\
\text { used to expand in vitro gland stem cells } \\
\text { that restored salivary function in murine } \\
\text { models of hyposalivation }{ }^{50,197}\end{array}$ & - \\
\hline \multicolumn{4}{|l|}{ Embryonic Organoids } \\
\hline $\begin{array}{l}\text { PRE-IMPLANTATION } \\
\text { MODELS } \\
\end{array}$ & MOUSE PSCS ${ }^{61}$ & - & - \\
\hline $\begin{array}{l}\text { EARLY POST- } \\
\text { IMPLANTATION } \\
\text { MODELS }\end{array}$ & $\begin{array}{c}\text { MOUSE PSCS }{ }^{62} \\
\text { HUMAN PSCS }{ }^{63,64}\end{array}$ & - & - \\
\hline GASTRULOIDS & $\begin{array}{c}\text { MOUSE PSCS } \\
\text { HUMAN PSCS } \\
\text { H7,68,70711}\end{array}$ & - & - \\
\hline NEURAL TUBE & MOUSE PSCS $\mathrm{S}^{72,73}$ & - & Growth in chemic ally-defined synthetic 3D matric es $\mathrm{s}^{73}$ \\
\hline
\end{tabular}

Abbreviations: PSCs - Pluripotent Stem Cells; ASCs - Adult Stem Cells; PLGA - poly(lactide-co-glycolide); HUVEC - Human Umbilical Vein Endothelial Cells, CFTR - Cystic fibrosis transmembrane conductance regulator. 


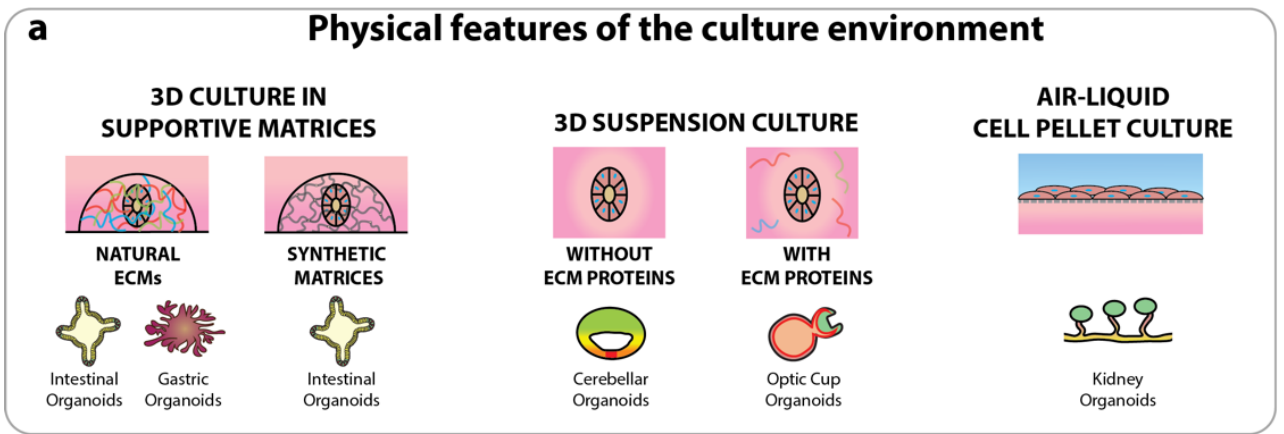

b Starting cell conditions and stages in organoid derivation
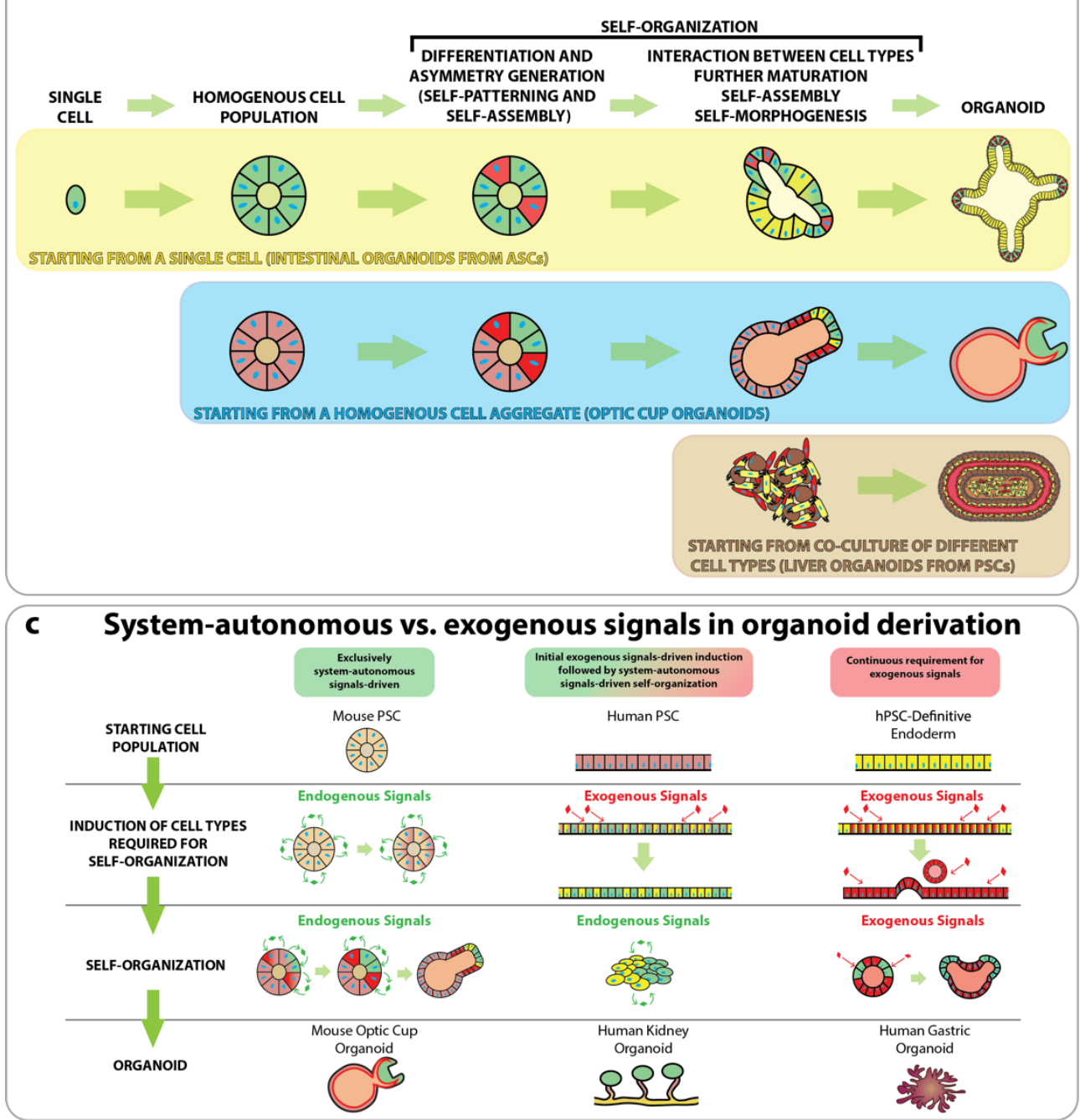

Fig. 1 | Overview of organoid derivation methods. a | The physical features of the culture environment are important to organoid development. The main types of culture environments are shown, together with examples of organoids that rely on them. $b$ | Organoids can be formed from cell populations with different starting conditions. Examples of an organoid protocol starting from a single cell (intestinal organoids), a homogeneous multicellular ensemble (optic cup organoids) and a heterogeneous co-culture of different cell types (liver organoids) are shown. The initial conditions are aligned with different steps of organoid development. ASC, adult stem cell; ECM, extracellular matrix; PSC, pluripotent stem cell. c | The formation of a specific organoid depends on different degrees of system-autonomous endogenous signals and exogenously supplied signals. Mouse optic cup formation depends largely on endogenous signals (left). Derivation of kidney organoids initially relies on exogenous signals that induce the ureteric epithelium and the metanephric mesenchyme from human pluripotent stem cells (hPSCs); subsequent self-organization steps rely on endogenous signals (centre). The supply of specific exogenous signals is continuously required for hPSC-derived gastric organoids (right). The necessity for exogenous signals likely substitutes for missing cell types that are responsible for the production of these signals during in vivo organogenesis. 




Fig. 2 | Starting cell type determines organoid maturation level in vitro. The graph shows the level of organoid maturation (vertical axis) that can be achieved according to the cells of origin (horizontal axis). Cancer cell-derived tumouroids, which show adult neoplastic phenotypes, should be considered a special case. PSCs, pluripotent stem cells

a) Basic Research
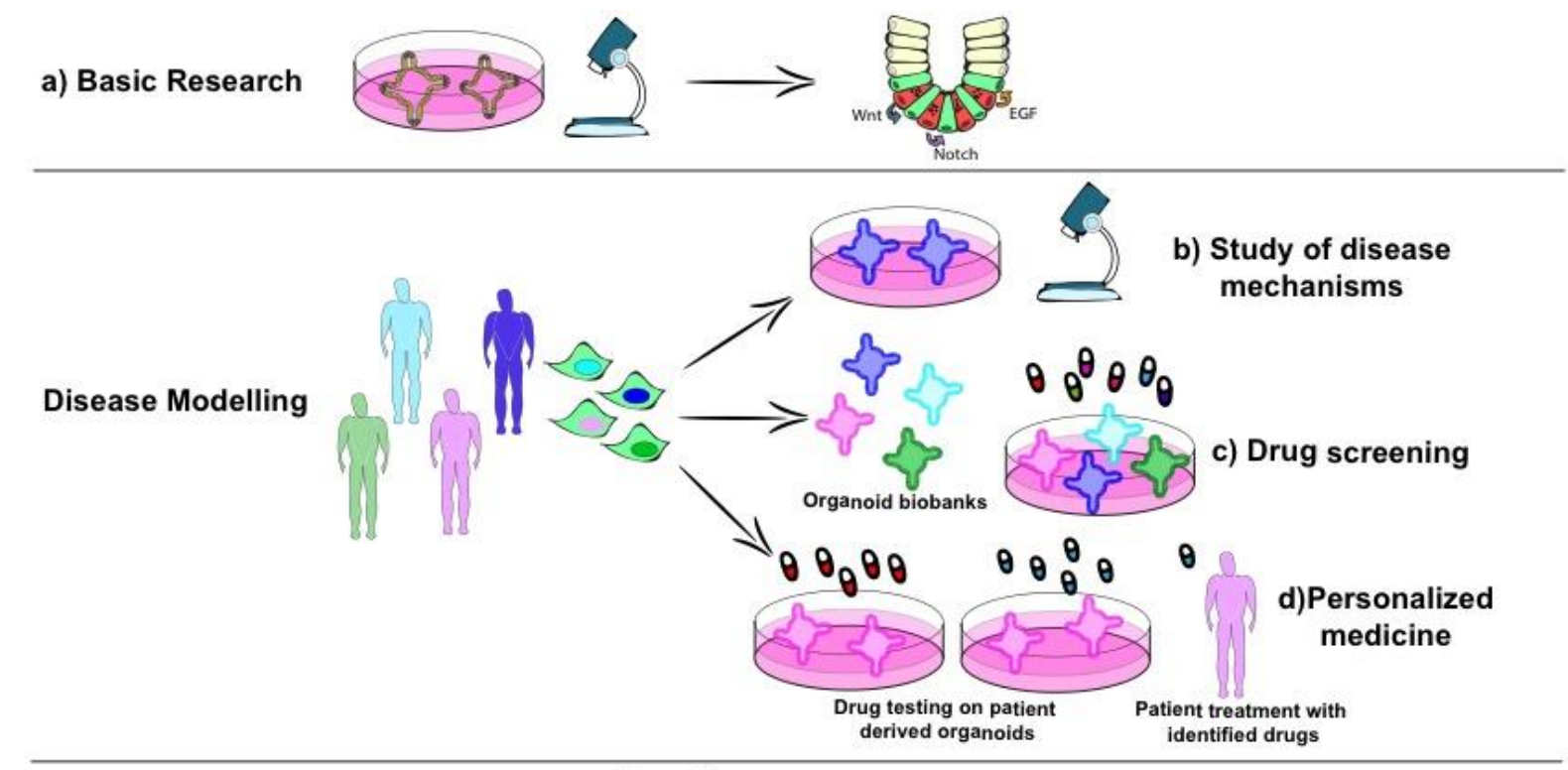

\section{e) Regenerative Medicine}
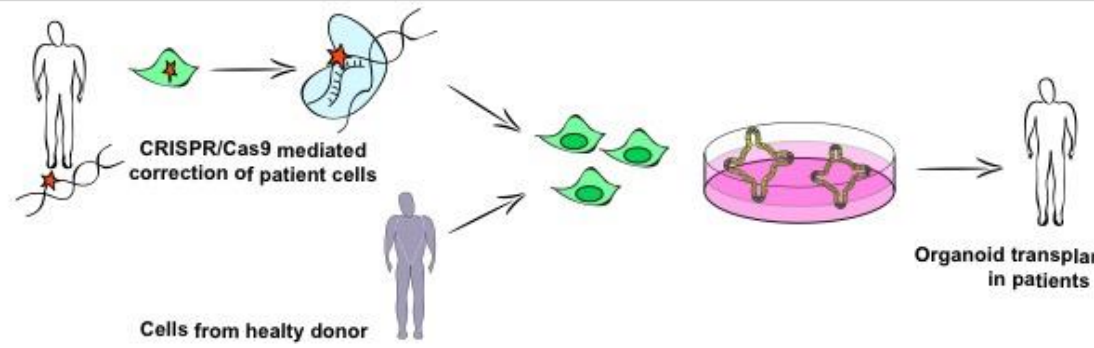

Organoid transplantation in patients

Fig. 3 | Key organoid applications. a | In basic research, organoids can be used to understand the principles of development, homeostasis and regeneration. $\mathrm{b}$ | Organoids derived from patient stem cells represent useful tools for the study of disease mechanisms because they replicate the complexity of the in vivo disease phenotype while still retaining the accessibility of in vitro systems. c | Organoid biobanks could be used to identify drugs effective against a broad spectrum of disease phenotypes. d | In personalized medicine, patient-specific organoids can help identify the best drug for each patient. e | Organoids derived from healthy donor cells, or from patients after genetic correction, can be used as a source of cells or 
tissues for regenerative medicine purposes (the red star represents a pathological genetic mutation). EGF, epidermal growth factor.

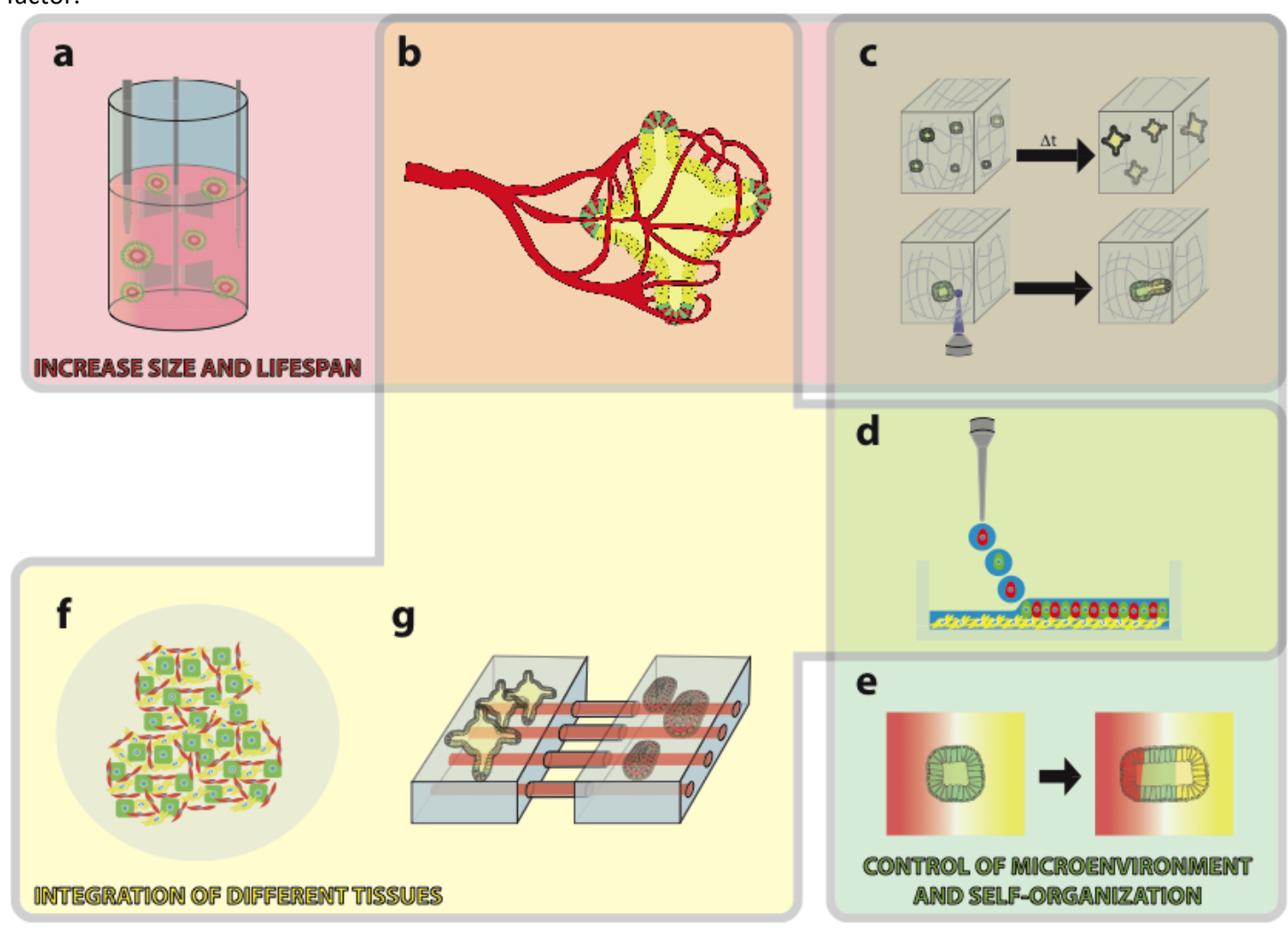

Fig. 4 | Bioengineering approaches to overcome key limitations of existing organoids. a | Bioreactors increase nutrient availability, which is usually limited by organoid size. b | The implementation of vascular networks in organoid culture could provide a near-physiological way to increase nutrient availability. $c$ | The control of the extracellular environment provides ways to steer organoid growth and self-organization towards a desired architecture. Synthetic materials with timedependent (top) or spatiotemporally programmable (bottom) mechanical and biochemical properties are promising tools for this purpose. d | The controlled spatial arrangement of different stem and/or progenitor cells may allow for defined reciprocal intercellular interactions, extending control over organoid self-organization. e | Following principles of embryonic development, the control of cell differentiation in space and time can be directed in vitro by the implementation of signalling centres and concentration gradients of developmentally relevant molecules (morphogens). $f \mid$ The co-culture of different cell types could allow for the formation and possibly the spatial self-organization of diverse tissues within a single organoid. g | Organoids could be integrated with ad hoc organ-on-chip technologies, allowing the connection and communication between multiple pre-formed 'organs'.

\section{Glossary}

Organoids. Stem cell-derived or progenitor cell-derived 3D structures that, on much smaller scales, re-create important aspects of the 3D anatomy and multicellular repertoire of their physiological counterparts and that can recapitulate basic tissue-level functions.

Adult stem cells(ASCs). Somatic stem cells with the capacity to give rise to the terminally differentiated cells of the tissue in which they reside.

Pluripotent stem cells (PSCs). Stem cells that have the capacity to give rise to all the cell types of the embryo proper. PSCs include embryonic stem cells and induced PSCs.

Organ-on-chip. Microfluidic cell culture device that contains perfused chambers in which living cells are arranged to simulate tissue-level and organ-level physiology. 
Symmetry-breaking. The first identifiable event that triggers the formation of an asymmetric system from an initially homogeneous (symmetric) state.

Reaction-diffusion mechanisms. Mathematical models that describe the dynamics of pattern formation in terms of local production and diffusion of activators and inhibitors and their interactions.

Bistability of regulatory networks. The behaviour of biological systems in which positive and negative feedback between networks of regulatory elements results in only one of two mutually exclusive outcomes.

Asymmetric cell division. The mechanism of cellular division by which the mother cell produces two daughter cells with different cell fates.

Air-liquid interface. A method for cell culture that is typically used for epithelial cells and in which a porous filter is used to expose basal cell layers to the cell culture medium and external cell layers to the air.

Morphogens. Biomolecules that act in a concentration-dependent manner to determine cell fate choices and tissue patterning in vivo.

Neuroepithelium (NE). Derived from neuroectoderm, the ectodermal embryonic compartment that will give rise to the nervous system. From this epithelium, components of the sensory organs are also formed, as in the case of the eye. Can be considered a synonym of neuroectoderm.

Neural retina (NR). A multi-layered structure of the eye formed by the neuronal types responsible for light acquisition and conversion into neural signals.

Retinal pigmented epithelium (RPE). A pigmented epithelium attached to the outside of the neural retina in the eye. It provides nutritional support to neural retina cells.

Ureteric epithelium. Derivative of the intermediate mesoderm that contributes to the formation of the renal collecting ducts.

Metanephric mesenchyme. Derivative of the intermediate mesoderm that substantially contributes to the formation of the renal nephrons.

Antral. The identity of the gastric epithelium in the most distal part of the stomach, pertaining to an anatomical region that connects to the intestinal duodenum called the pyloric antrum.

Fundic. The identity of the gastric epithelium in the upper

curvature of the stomach, pertaining to an anatomical region called the fundus of the stomach. 
Mesodermal. Derived from the mesoderm, the middle embryonic germ layer that is formed upon gastrulation and will give rise to different adult tissues such as skeletal and cardiac muscle, smooth muscle, blood, cartilage, bones and dermis.

Surface ectoderm. The part of the embryonic ectoderm that lines the exterior of the embryo (the surface). Derivatives of the surface ectoderm include the epidermis with associated glands (including the mammary glands), sensory receptors, the epithelium of the oral and nasal cavity with associated glands and the cornea and the lens of the eye, among others.

Glandular tissues. Epithelial tissues that produce and release biomolecules (hormones and growth factors, among others) into the bloodstream, in external or internal body cavities.

Endodermal. Derived from the endoderm, the most inner embryonic germ layer formed upon gastrulation, that will give rise to internal structures such as the epithelial lining of the respiratory and gastrointestinal tracts and of the urinary system.

Gastrulation. A process that occurs during mammalian embryonic development in which the three germ layers (ectoderm, mesoderm and endoderm) form from the epiblast and arrange in space to generate a more defined body plan of the organism.

Enterospheres. Spherical epithelial cysts of fetal intestinal progenitors.

Crypts. Invaginations of the intestinal epithelium at the base of villi, which typically host selfrenewing LGR5+ intestinal stem cells and Paneth cells at their bottom.

Optic vesicles. Embryonic epithelial vesicles evaginating from the forebrain neuroepithelium that will give rise to the optic cup. In organoids, they are the vesicular structures forming at early stages of organoid development, before the morphological changes that will give rise to the final optic cup structure.

Personalized medicine. A medical procedure in which the therapy and/or treatment is tailored to a specific patient.

Organoid biobanks. Collections of organoid samples, typically of human origin, that are stored for research purposes and aim to encompass a wide range of population genetic variance.

$3 R$ principles. A framework for more humane animal research aimed at replacing, reducing and refining animal experimentation.

Bioreactors. Devices for the large-scale expansion of cells under controlled culture conditions.

Sacrificial moulds. Templates used to form a structure; they are dissolved after the moulding process.

Laser ablation. The controlled removal of portions of a material through irradiation with a laser beam. 
Bioprinting. The use of specialized 3D printing technologies to combine cells, biomolecules and biomaterials in 3D assemblies.

Biosensors. Devices comprising biological components (such as antibodies and enzymes, among others) and electrochemical components for the measurement of biological parameters.

\section{Acknowledgements}

The work of M.P.L. in the area of organoid biology and technology is supported by the Swiss National Science Foundation, the European Union's Horizon 2020 research and innovation programme (INTENS 668294), the Personalized Health and Related Technologies Initiative from the ETH Board, the Vienna Science and Technology Fund and École Polytechnique Fédérale de Lausanne (EPFL). The authors are grateful to their collaborators in the organoid field, including J. Briscoe, H. Clevers, D. Duboule, A. Grapin-Botton, A. Kitcheva, J. Knoblich, A. Martinez-Arias and E. Tanaka. The authors thank the members of their laboratory for helpful discussions and apologize to all the scientists whose work they could not cite owing to space restrictions.

\section{Author contributions}

G.R., A.M. and M.P.L. researched data for the article, made substantial contributions to discussions of the content and wrote the article. M.P.L. reviewed and/or edited the manuscript before submission.

\section{Competing interests}

The authors declare no competing interests. 